\title{
APPENDIX I. METHODS FOR LABORATORY-MEASURED PHYSICAL PROPERTIES, GEARHART-OWEN WELL LOGS, AND THE UYEDA DOWN-HOLE TEMPERATURE PROBE, LEG 61, DEEP SEA DRILLING PROJECT ${ }^{1}$
}

\author{
Robert E. Boyce, Scripps Institution of Oceanography, Deep Sea Drilling Project, La Jolla, California
}

\section{LABORATORY-MEASURED PHYSICAL PROPERTIES}

\section{Introduction}

In the laboratory, we measured heat conductivity, sound velocity, 2-minute GRAPE wet-bulk density, gravimetric wet-bulk density, wet-water content, and porosity on the same sample, when possible.

An attempt was made to obtain an undisturbed sample. This is a serious problem in the upper 200 meters of the hole, where the sediments are soft, as the drill bit is $25 \mathrm{~cm}$ in diameter, with the $\sim 6$-cm-diameter coring hole within this cutting bit. Most soft sediments are literally "squirted" into the core barrel and completely disturbed.

Because of this core disturbance, few discrete samples for physical-property measurements were taken in the upper part of the hole; however, the continuousanalog GRAPE was always run. Also as a result of the degree of core disturbance in the upper part of the hole, and stiffness of samples which were not disturbed, vane shear strength could be measured on only a small number of "semi-soft" samples.

The criterion for an undisturbed sample is undistorted bedding or laminae in the sample. The obvious problem with this criterion is that a completely homogeneous soft-sediment core would not be sampled, even if it were undisturbed.

In general, sediment remained in the core liner for about 2 hours. The core section was split, and samples were selected immediately for heat-conductivity measurement, which was done immediately (2-5 minutes). Then a D-shaped sample was cut for the sound-velocity measurement, and a smear slide was made; this sample was wrapped in plastic and placed in a small plastic box with a wet sponge. The sample was then removed from the plastic wrapper, and its surface cleaned with either a spatula or file. Distilled water was squirted on these surfaces, and the velocities were measured; then the GRAPE 2-minute wet-bulk-density determination was made. Afterward, a sub-sample was cut for gravimetric determination of wet-water content, wet-bulk density, and porosity; this sample was cleaned and wrapped in plastic, then sealed in a plastic vial with a wet towel. The vial was placed in a refrigerator above freezing until it could be processed.

\footnotetext{
${ }^{1}$ Initial Reports of the Deep Sea Drilling Project, Volume 61.
}

Basalt samples were handled differently, because of DSDP's official curatorial process. Basalt was not in a plastic liner; therefore, it was necessary to select soundvelocity samples when the cores arrived on deck and were brought into the laboratory. These core segments were wrapped in plastic until the samples were processed by the DSDP curator and eventually split. After the core segment was split, heat conductivity was measured immediately ( $2-5$ minutes) on the split core. Then a minicore $(\sim 2 \mathrm{~cm}$ diameter) was drilled out of this $1 / 2$ core segment. This mini-core was wrapped in plastic and placed in a plastic vial with a damp sponge until velocity and a 2-minute GRAPE were measured. Velocity was measured across the axis of the mini-core, and 2-minute GRAPE was measured parallel to the axis of the minicore. The mini-core was then wrapped in plastic and sealed in a vial with a wet sponge and placed in a refrigerator (slightly above freezing) until its wet-bulk density, wet-water content, and porosity were gravimetrically determined.

A variation on the basalt sampling method was as follows: in some cases we measured the velocity of the basalt when the core first arrived on deck. The velocity of the basalt was measured across the $6.6-\mathrm{cm}$ diameter of the core after filing and wetting with distilled water. This core sequence was then wrapped with plastic until curatorial procedures were completed and it was split. The remaining physical properties were measured as discussed in the mini-core method. This method gives better and significantly higher velocities than the mini-core method. This is true because (1) sample size is larger, which reduces the error, and (2) the sample will never be more saturated with water than at that time.

The mini-core method was used at Hole 462 in Cores 60 through 66 , and the full-core-diameter method was used on Cores 66 through 69 . At Hole 462A, the fullcore-diameter method was used from Core 15 through Core 74. From Core 75 to 92 , new shipboard physicalproperty scientists, who were not satisfied with the whole-core-diameter method, again used the mini-core technique; occasionally velocity was measured across the radius of a split core. Because of these different techniques, it will not be possible to precisely compare all these data, as in scatter diagrams, etc.

\section{Heat Conductivity}

Heat conductivity is the quantity of heat per second which will flow between opposite faces of a cube when there is a $1^{\circ} \mathrm{C}$ temperature difference between opposite 
faces of the cube. For a 1-cm cube, heat conductivity is a coefficient in units of $\mathrm{cal} \cdot 10^{-3} /\left(\mathrm{cm} \cdot{ }^{\circ} \mathrm{C} \cdot \mathrm{s}\right)$.

Heat conductivity was measured with a Quick Thermal Conductivity Meter (QTM). This device uses a rectangular pad with a heater and thermocouple, which is placed on a flat rock sample, ${ }^{2}$ and the thermal conductivity is "automatically" measured (over a 20-second period; thus, drift is small) calculated, and displayed on a panel. The device has known standards which are run with measurements. Deviation from these standards is slight, and correction factors (Tables 1, 2, and 3) are applied to all conductivities after they are adjusted to $21^{\circ} \mathrm{C}$ (Table 4). The principle is that described in Von Herzen and Maxwell (1969), except that the heater and thermocouple are between a pad (on the surface of the split core) and the rock; in the Von Herzen and Maxwell (1969) technique, the needle is centered in a complete, round sediment core. Because the needle is placed on the sample surface then, the formula used by Von Herzen and Maxwell (1969) is divided in half and a new empirical correction factor is inserted that empirically calibrates the data for the probe, etc.

However, to be certain that we were getting good data, we ran as many standards as possible and, in addition, inserted our own correction factor. One of the reasons for doing this is that the only working needle pad was not waterproof; therefore, we inserted a thin film of plastic between the needle pad and flat rock surface.

The conductivity standards are accurate only to $\pm 5 \%$ and the precision of the $\mathrm{QTM}^{\mathrm{R}}$ data is $\pm 10 \%$. All raw thermal-conductivity data are presented in Table 4;

\footnotetext{
${ }^{2}$ The thermal conductivity pad is of very soft, pliable, cheesecloth-like fibers; the heating element is a -2 -mm-wide, pliable metal foil. A very thin pliable film of plastic was placed over the water-saturated sample. A weight on the soft pad molded the heating foil and pad fibers; therefore, any error resulting from a slightly irregular surface to the slightly irregular sample surface is probably relatively small compared to the $\pm 10 \%$ precision of the QTM. However, future testing should be done to verify this assumption. The pad was placed on the sample at least 2 minutes prior to measurement.
}

the temperature of the core was estimated from the time elapsed between the time of measurement and the time the core arrived on deck. This was done by using Figure 1 , which is a plot of temperature of water in a core liner versus time, as it rises for $\sim 2$ to $\sim 24^{\circ} \mathrm{C}$ while setting at room temperature $\left(\sim 24^{\circ} \mathrm{C}\right)$ for 5 hours. This curve should be the maximum time required by the cores. On Core 36 of Hole 462, we measured the temperature of the soft core and noted the time elapsed after the core arrived on deck. Therefore, by entering this temperature into the temperature-time curve of Figure 1, we can extrapolate backward to that time when the core arrived on deck. By this technique, we found the temperature of the core to be about $14.2^{\circ} \mathrm{C}$ when it arrived on deck. Therefore, knowing this temperature and knowing what time had elapsed before the thermal-conductivity measurement, we could use the upper time axis in Figure 1 to extrapolate this amount of time from $14.2^{\circ} \mathrm{C}$ on Figure 1 forward through time to find the temperature of the core during the thermal-conductivity measurements and sound-velocity measurements.

All assumptions, adjustments and correction factors are in Table 4. Temperature corrections to $21^{\circ} \mathrm{C}$ assume a $+1 \%$ change in thermal conductivity for every $+4^{\circ} \mathrm{C}$ change in temperature (Clark, 1966). This is typical of water and is used here for simplicity. The temperature of the thermal-conductivity measurement was the core temperature plus the average temperatures during the red and green lights. The red and green lights indicate the beginning and end of the thermal-conductivity measurement, and these temperatures were measured from the QTM machine after the QTM was zeroed at core temperature.

The conductivity data from measurements on standards were corrected to $21^{\circ} \mathrm{C}$ before calculating correction factors (Tables 1-3). All thermal-conductivity data were corrected to $21^{\circ} \mathrm{C}$ before multiplying by the appropriate correction factor derived from the stand-

Table 1. Leg 61 QTM thermal-conductivity values for the $1.16\left(\mathrm{kcal} / \mathrm{mh}^{\circ} \mathrm{C}\right)$ standard, while the QTM is set at $20 \mathrm{sec}$, heater $=8$, and mode $=$ high .

\begin{tabular}{|c|c|c|c|c|c|c|c|c|}
\hline \multirow[b]{2}{*}{ Date } & \multicolumn{3}{|c|}{ QTM Settings } & \multirow{2}{*}{$\begin{array}{c}\text { Room } \\
\text { Temperature } \\
\left({ }^{\circ} \mathrm{C}\right)\end{array}$} & \multirow{2}{*}{$\begin{array}{l}\text { Average of } \\
\text { Red and Green } \\
\text { Temperatures } \\
\Delta \mathrm{T} \\
\left({ }^{\circ} \mathrm{C}\right)\end{array}$} & \multirow{2}{*}{$\begin{array}{l}\text { Temperature of } \\
\text { Measurement } \\
\text { (room temp. } \\
\quad+\Delta \mathrm{T}) \\
\left({ }^{\circ} \mathrm{C}\right)\end{array}$} & \multirow{2}{*}{$\begin{array}{l}\text { QTM } \\
\begin{array}{c}\text { Measurement } \\
\text { of Standard }\end{array} \\
\text { Conductivity } \\
\left(\mathrm{kcal} / \mathrm{mh}^{\circ} \mathrm{C}\right)\end{array}$} & \multirow{2}{*}{$\begin{array}{l}\text { Theoretical } \\
\text { Conductivity } \\
\text { Value of } \\
\text { Standard } \\
\left(\text { (kcal/mh } / \mathrm{mb}^{\circ} \mathrm{C}\right.\end{array}$} \\
\hline & $\begin{array}{c}\text { Time } \\
(s)\end{array}$ & Heater & Mode & & & & & \\
\hline 9 July 1978 & 20 & 8 & High & 23 & $(21+24) / 2=22.5$ & 45.5 & 1.149 & 1.16 \\
\hline 9 July 1978 & 20 & 8 & High & 23 & $(22+24) / 2=23.0$ & 46 & 1.179 & 1.16 \\
\hline 9 July 1978 & 20 & 8 & High & 23 & $(21+24) / 2=22.5$ & 45.5 & 1.220 & 1.16 \\
\hline 9 July 1978 & 20 & 8 & High & 23 & $(20.5+23) / 2=21.75$ & 44.75 & 1.190 & 1.16 \\
\hline 9 July 1978 & 20 & 8 & High & 23 & $(21+24) / 2=22.5$ & 45.5 & 1.212 & 1.16 \\
\hline 8 July 1978 & 20 & 8 & High & 24 & $(22+24) / 2=23.0$ & 47 & 1.181 & 1.16 \\
\hline 8 July 1978 & 20 & 8 & High & 24 & $(22+25) / 2=23.5$ & 47.5 & 1.189 & 1.16 \\
\hline 8 July 1978 & 20 & 8 & High & 24 & $(22+25) / 2=23.5$ & 47.5 & 1.199 & 1.16 \\
\hline 8 July 1978 & 20 & 8 & High & 24 & $(21+24) / 2=22.5$ & 46.5 & 1.203 & 1.16 \\
\hline 8 July 1978 & 20 & 8 & High & 24 & $(24+22) / 2=23.0$ & 47.0 & 1.207 & 1.16 \\
\hline \multicolumn{9}{|c|}{$\begin{array}{l}\text { Notes: Number of Samples }=10 \text {. } \\
\text { Mean at } 45^{\circ} \mathrm{C}=1.193 \mathrm{kcal} / \mathrm{mh}^{\circ} \mathrm{C} \text { at } \sim 45^{\circ} \mathrm{C} \text {. } \\
\text { Mean at } 21^{\circ} \mathrm{C}=1.121 \mathrm{kcal} / \mathrm{mh}^{\circ} \mathrm{C} \text { at }-21^{\circ} \mathrm{C} \text { (temperature correction }=-0.25 \% \text { per }{ }^{\circ} \mathrm{C} \text { ). } \\
\text { Range }=+2.3 \%,-3.7 \% \text {. }\end{array}$} \\
\hline \multicolumn{9}{|c|}{ Empirical Correction Factor $=K=\frac{1.1339 \mathrm{kcal} / \mathrm{mh}^{\circ} \mathrm{C}}{1.12 \mathrm{kcal} / \mathrm{mh}^{\circ} \mathrm{C}}=1.0124$} \\
\hline \multicolumn{9}{|c|}{$K=$ true conductivity at $21^{\circ} \mathrm{C}$} \\
\hline \multicolumn{9}{|c|}{$K=\overline{\text { measured conductivity at } 21^{\circ} \mathrm{C}}$} \\
\hline \multicolumn{9}{|c|}{ 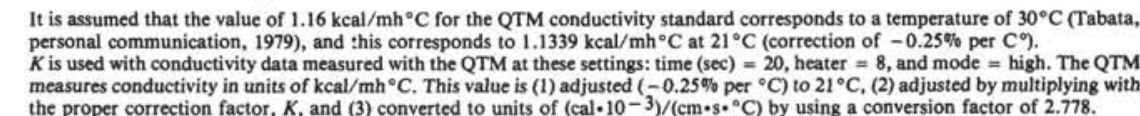 } \\
\hline
\end{tabular}


Table 2. Leg 61 thermal conductivity standard values for the $1.16\left(\mathrm{kcal} / \mathrm{mh}^{\circ} \mathrm{C}\right)$ standard, while the QTM is set at $20 \mathrm{sec}$, heater $=4$, and mode $=$ high.

\begin{tabular}{|c|c|c|c|c|c|c|c|c|c|c|}
\hline \multirow[b]{2}{*}{ Hole } & \multirow[b]{2}{*}{ Core } & \multicolumn{3}{|c|}{ QTM Settings } & \multirow{2}{*}{$\begin{array}{c}\text { Room } \\
\text { Temperature } \\
\left({ }^{\circ} \mathrm{C}\right)\end{array}$} & $\begin{array}{l}\text { Average of } \\
\text { Red and Green } \\
\text { Temperatures }\end{array}$ & $\begin{array}{l}\text { Temperature of } \\
\text { Measurement } \\
\text { (room temp. }\end{array}$ & $\begin{array}{c}\text { QTM } \\
\text { Measurement } \\
\text { of Standard }\end{array}$ & $\begin{array}{l}\text { Theoretical } \\
\text { Conductivity } \\
\text { Value of }\end{array}$ & \\
\hline & & $\begin{array}{l}\text { Time } \\
\text { (sec) }\end{array}$ & Heater & Mode & & $\begin{array}{l}\Delta \mathrm{T} \\
\left({ }^{\circ} \mathrm{C}\right)\end{array}$ & $\begin{array}{l}+\Delta \mathrm{T} \\
\left({ }^{\circ} \mathrm{C}\right)\end{array}$ & $\begin{array}{l}\text { Conductivity } \\
\text { (kcal/mh }{ }^{\circ} \mathrm{C} \text { ) }\end{array}$ & $\begin{array}{l}\text { Standard } \\
\left(\mathrm{kcal} / \mathrm{mh}^{\circ} \mathrm{C}\right)\end{array}$ & Date \\
\hline - & - & 20 & 4 & High & 23.5 & $(10 ?+11.5) / 2=10.75$ & 34.25 & 1.136 & 1.16 & 28 May 1978 \\
\hline - & - & 20 & 4 & High & 23.5 & $(9 ?+11) / 2=10$ & 33.5 & 1.117 & 1.16 & 28 May 1978 \\
\hline - & - & 20 & 4 & High & 23.5 & $(10 ?+11.3) / 2=10.75$ & 34.25 & 1.132 & 1.16 & 28 May 1978 \\
\hline - & - & 20 & 4 & High & 23.5 & $(10 ?+12) / 2=11$ & 34.5 & 1.172 & 1.16 & 28 May 1978 \\
\hline- & - & 20 & 4 & High & 23.5 & $(10 ?+12) / 2=11$ & 34.5 & 1.182 & 1.16 & 28 May 1978 \\
\hline - & - & 20 & 4 & $\mathrm{High}$ & 23.5 & $(10 ?+12) / 2=11$ & 34.5 & 1.169 & 1.16 & 28 May 1978 \\
\hline - & - & 20 & 4 & High & 23.5 & $(10 ?+12) / 2=11$ & 34.5 & 1.168 & 1.16 & 28 May 1978 \\
\hline- & - & 20 & 4 & High & 23.5 & $(10 ?+12) / 2=11$ & 34.5 & 1.161 & 1.16 & 28 May 1978 \\
\hline - & - & 20 & 4 & High & 23.5 & $(10 ?+12) / 2=11$ & 34.5 & 1.158 & 1.16 & 28 May 1978 \\
\hline - & - & 20 & 4 & High & 23.5 & $(10 ?+11.3) / 2=10.75$ & 34.25 & 1.154 & 1.16 & 28 May 1978 \\
\hline - & - & 20 & 4 & High & 24 & $(12+13) / 2=12.5$ & 36.5 & 1.130 & 1.16 & 8 July 1978 \\
\hline - & - & 20 & 4 & High & 24 & $(11+13) / 2=12$ & 36 & 1.100 & 1.16 & 8 July 1978 \\
\hline - & - & 20 & 4 & High & 24 & $(11+12.5) / 2=11.75$ & 35.75 & 1.100 & 1.16 & 8 July 1978 \\
\hline - & - & 20 & 4 & High & 24 & $(11+12.5) / 2=11.75$ & 35.75 & 1.107 & 1.16 & 8 July 1978 \\
\hline - & - & 20 & 4 & High & 24 & $(11+12.5) / 2=11.75$ & 35.75 & 1.099 & 1.16 & 8 July 1978 \\
\hline - & - & 20 & 4 & High & 23 & $(16+18) / 2=17$ & 40 & 1.092 & 1.16 & 9 July 1978 \\
\hline - & - & 20 & 4 & High & 23 & $(11.5-13) / 2=12.75$ & 35.25 & 1.119 & 1.16 & 9 July 1978 \\
\hline - & - & 20 & 4 & High & 23 & $(11-12.5) / 2=11.75$ & 34.75 & 1.100 & 1.16 & 9 July 1978 \\
\hline - & - & 20 & 4 & High & 23 & $(12+13) / 2=12.5$ & 35.5 & 1.094 & 1.16 & 9 July 1978 \\
\hline - & - & 20 & 4 & High & 23 & $(11+12.5) / 2=11.75$ & 34.75 & 1.074 & 1.16 & 9 July 1978 \\
\hline 462 & 14 & 20 & 4 & High & 23.5 & 12 & 33.5 & 1.141 & 1.16 & - \\
\hline 462 & 16 & 20 & 4 & High & 23.5 & 12 ? & 33.5 ? & 1.186 & 1.16 & - \\
\hline 462 & 28 & 20 & 4 & High & 24 & 12 ? & $34 ?$ & 1.135 & 1.16 & - \\
\hline 462 & 37 & 20 & 4 & High & 24 & 13.5 & $34.25 ?$ & 1.148 & 1.16 & - \\
\hline 462 & 39 & 20 & 4 & High & 24.5 & 13.5 ? & $34.25 ?$ & 1.150 & 1.16 & - \\
\hline 462 & 48 & 20 & 4 & High & 24 & 13.5 ? & $34.25 ?$ & 1.213 & 1.16 & - \\
\hline 462 & 49 & 20 & 4 & High & 24 & 11.0 & 33.5 & 1.140 & 1.16 & - \\
\hline 462 & 50 & 20 & 4 & High & 24 & 11.5 & 35.5 & 1.14 & 1.16 & - \\
\hline 462 & 51 & 20 & 4 & High & 24 & 10.5 & 34.5 & 1.139 & 1.16 & - \\
\hline 462 & 52 & 20 & 4 & High & 24 & $10.5 ?$ & $34.5 ?$ & 1.155 & 1.16 & - \\
\hline 462 & 55 & 20 & 4 & High & 24 & 10.5? & $34.5 ?$ & 1.155 & 1.16 & - \\
\hline 462 & 57 & 20 & 4 & High & 24 & $10.5 ?$ & $34.5 ?$ & 1.136 & 1.16 & - \\
\hline 462 & 58 & 20 & 4 & High & 24 & $10.5 ?$ & $34.5 ?$ & 1.154 & 1.16 & - \\
\hline 462 & 59 & 20 & 4 & High & 24 & 10.5 ? & $34.5 ?$ & 1.136 & 1.16 & - \\
\hline 462 & 60 & 20 & 4 & High & 24 & $10.5 ?$ & $34.5 ?$ & 1.133 & 1.16 & - \\
\hline 462 & 64 & 20 & 4 & High & 24 & 11.0 & 35 & 1.130 & 1.16 & - \\
\hline 462 & 65 & 20 & 4 & High & 24 & 10.5 & 34.5 & 1.159 & 1.16 & - \\
\hline 462 & 67 & 20 & 4 & High & 24 & 11.0 & 35 & 1.135 & 1.16 & - \\
\hline 462 & 68 & 20 & 4 & High & 24 & 11.0 & 35.0 & 1.265 & 1.16 & - \\
\hline 462 & 69 & 20 & 4 & High & 23 & 12.5 & 35.5 & 1.179 & 1.16 & - \\
\hline $462 \mathrm{~A}$ & 7 & 20 & 4 & High & 24 & 12.5 ? & 35.5 ? & 1.115 & 1.16 & - \\
\hline $462 \mathrm{~A}$ & 8 & 20 & 4 & High & 24 & 12.5 ? & 35.5 ? & 1.194 & 1.16 & - \\
\hline $462 \mathrm{~A}$ & $\mathrm{H}-4$ & 20 & 4 & High & 24 & 12 & 36 & 1.103 & 1.16 & - \\
\hline $462 \mathrm{~A}$ & 9 & 20 & 4 & High & 24 & 12 & 36 & 1.084 & 1.16 & - \\
\hline $462 \mathrm{~A}$ & 17 & 20 & 4 & High & 24 ? & 12 ? & 36 & 1.094 & 1.16 & - \\
\hline $462 \mathrm{~A}$ & 18 & 20 & 4 & High & 24 & 12 & 36 & 1.104 & 1.16 & - \\
\hline $462 \mathrm{~A}$ & 28 & 20 & 4 & High & 24 & 13 & 37 & 1.096 & 1.16 & - \\
\hline $462 A$ & 32 & 20 & 4 & High & 24 & $13 ?$ & $37 ?$ & 1.075 & 1.16 & - \\
\hline $462 A$ & 38 & 20 & 4 & High & 24 & 13 ? & $37 ?$ & 1.089 & 1.16 & - \\
\hline $462 A$ & 39 & 20 & 4 & High & 24 & 13 ? & $37 ?$ & 1.038 & 1.16 & - \\
\hline $462 \mathrm{~A}$ & 40 & 20 & 4 & High & 24 & 13 ? & $37 ?$ & 1.116 & 1.16 & - \\
\hline $462 \mathrm{~A}$ & 45 & 20 & 4 & High & 23 & 13 ? & $37 ?$ & 1.019 & 1.16 & - \\
\hline $462 \mathrm{~A}$ & 46 & 20 & 4 & High & 24 & 13 ? & $37 ?$ & 1.118 & 1.16 & - \\
\hline $462 \mathrm{~A}$ & 51 & 20 & 4 & High & 24 & 13 ? & $37 ?$ & 1.125 & 1.16 & - \\
\hline $462 \mathrm{~A}$ & 52 & 20 & 4 & High & 24 & 12 & 36 & 1.101 & 1.16 & - \\
\hline $462 \mathrm{~A}$ & 59 & 20 & 4 & High & 23 & 11.0 & 34 & 1.110 & 1.16 & - \\
\hline $462 \mathrm{~A}$ & 61 & 20 & 4 & High & 24 & 12 & 36 & 1.123 & 1.16 & - \\
\hline $462 \mathrm{~A}$ & 62 & 20 & 4 & High & 24 & 12 & 36 & 1.110 & 1.16 & - \\
\hline $462 \mathrm{~A}$ & 64 & 20 & 4 & High & 24 & 12 & 36 & 1.137 & 1.16 & - \\
\hline $462 \mathrm{~A}$ & 65 & 20 & 4 & High & 24 & 11 & 35 & 1.091 & 1.16 & - \\
\hline $462 \mathrm{~A}$ & 65 & 20 & 4 & High & 24 & 12 & 36 & 1.097 & 1.16 & - \\
\hline $462 \mathrm{~A}$ & 67 & 20 & 4 & High & 24 & 12 ? & $36 ?$ & 1.057 & 1.16 & - \\
\hline $462 A$ & 68 & 20 & 4 & High & 24 & 11.5 & 35.5 & 1.135 & 1.16 & - \\
\hline $462 \mathrm{~A}$ & 70 & 20 & 4 & High & 24 & 12 & 36 & 1.076 & 1.16 & - \\
\hline $462 \mathrm{~A}$ & 71 & 20 & 4 & High & 24 & 11.5 & 35.5 & 1.107 & 1.16 & - \\
\hline $462 \mathrm{~A}$ & 74 & 20 & 4 & High & 24 & 13.5 & 37.5 & 1.072 & 1.16 & - \\
\hline $462 \mathrm{~A}$ & 76 & 20 & 4 & High & 21.5 & i1. 5 ? & 33? & 1.166 & 1.16 & - \\
\hline $462 \mathrm{~A}$ & 77 & 20 & 4 & High & 21.5 & 11.s? & 33 ? & 1.152 & 1.16 & - \\
\hline $462 \mathrm{~A}$ & 78 & 20 & 4 & High & 21.5 & 11.5 ? & $33 ?$ & 1.154 & 1.16 & - \\
\hline $462 \mathrm{~A}$ & 79 & 20 & 4 & High & 21.5 & 11.5 ? & $33 ?$ & 1.161 & 1.16 & - \\
\hline $462 \mathrm{~A}$ & 80 & 20 & 4 & High & 21.5 & 11.5 ? & 33 ? & 1.160 & 1.160 & - \\
\hline $462 \mathrm{~A}$ & 81 & 20 & 4 & High & 21.5 & 11.5 ? & 33 ? & 1.160 & 1.160 & - \\
\hline $462 \mathrm{~A}$ & 84 & 20 & 4 & High & 21 & 12 ? & $33 ?$ & 1.154 & 1.160 & - \\
\hline $462 \mathrm{~A}$ & 85 & 20 & 4 & High & 22 & 11 ? & $33 ?$ & 1.155 & 1.16 & - \\
\hline $462 \mathrm{~A}$ & 87 & 20 & 4 & High & 22 & 11? & 33 ? & 1.154 & 1.16 & - \\
\hline $462 \mathrm{~A}$ & 88 & 20 & 4 & High & 22 & II? & 33 ? & 1.166 & 1.16 & - \\
\hline $462 \mathrm{~A}$ & 89 & 20 & 4 & High & 22 & i1? & 33 ? & 1.157 & 1.16 & - \\
\hline $462 \mathrm{~A}$ & 90 & 20 & 4 & High & 22 & 11 ? & 33 ? & 1.150 & 1.16 & - \\
\hline
\end{tabular}

Notes: Number of samples $=78$

Mean at $-35^{\circ} \mathrm{C}=1.131(\mathrm{kcal}) /\left(\mathrm{mh}^{\circ} \mathrm{C}\right)$

Mean at $-21^{\circ} \mathrm{C}=1.096\left(\mathrm{kcal} / \mathrm{mh}^{\circ} \mathrm{C}\right)$ (temperature correction $\left.=-0.25 \% \mathrm{per}{ }^{\circ} \mathrm{C}\right)$

Range $=+7.0 \%,-9.8 \%$

Empirical correction factor $=K_{1.16 \mathrm{STD}}=\frac{1.1339\left(\mathrm{kcal} / \mathrm{mhh}^{\circ} \mathrm{C}\right)}{1.096\left(\mathrm{kcal} / \mathrm{mh}^{\circ} \mathrm{C}\right)}=1.035$.

$K=\frac{\text { true conductivity at } 21^{\circ} \mathrm{C}}{\text { measured conductivity at } 21^{\circ} \mathrm{C}}$

It is assumed that the value of $1.16 \mathrm{kcal} / \mathrm{mhh}^{\circ} \mathrm{C}$ for the QTM conductivity standard corresponds to a temperature of $30^{\circ}(\mathrm{Tabata}$, personal communica-

tion, 1979), and this corresponds to $1.1339 \mathrm{kcal} / \mathrm{mh}^{\circ} \mathrm{C}$ at $21^{\circ} \mathrm{C}$ (correction of $-0.25 \%$ per ${ }^{\circ} \mathrm{C}$ ).
$\left(K_{0.196 \mathrm{STD}}+K_{1.16 \mathrm{STD}}\right) / 2=(1.030+1.035) / 2=1.0325=$ averaged $K$, which is used with the conductivity data measured with the QTM at these settings: time ( $\mathrm{sec})=20$, heater $=4$, and mode $=$ high. The QTM measures conductivity in units of $\mathrm{kcal} / \mathrm{mh}{ }^{\circ} \mathrm{C}$. This value is (1) adjusted $(-0.25 \%$ per ${ }^{\circ} \mathrm{C}$ ) to $21^{\circ} \mathrm{C},(2)$ adjusted by using correction factor, $K$, and (3) converted to units of (cal $\left.10^{-} 3\right) /\left(\mathrm{cm} \cdot \mathrm{sec} \cdot{ }^{\circ} \mathrm{C}\right)$ by using a conversion factor of
2.778 . 


\section{R. E. BOYCE}

Table 3. Leg 61 QTM thermal-conductivity values for the $0.196\left(\mathrm{kcal} / \mathrm{mh}^{\circ} \mathrm{C}\right)$ standard, while the QTM is set at $20 \mathrm{sec}$, heater $=4$, and mode $=$ high.

\begin{tabular}{|c|c|c|c|c|c|c|c|c|c|c|}
\hline Hole & Core & $\begin{array}{l}\text { Time } \\
\text { (sec) }\end{array}$ & rM Setti & Mode & $\begin{array}{c}\text { Room } \\
\text { Temperature } \\
\left({ }^{\circ} \mathrm{C}\right)\end{array}$ & $\begin{array}{c}\text { Average of } \\
\text { Red and Green } \\
\text { Temperatures } \\
\Delta \mathrm{T} \\
\left({ }^{\circ} \mathrm{C}\right)\end{array}$ & $\begin{array}{l}\text { Temperature of } \\
\text { Measurement } \\
\text { (room temp. } \\
+\Delta \mathrm{T} \\
\left({ }^{\circ} \mathrm{C}\right)\end{array}$ & $\begin{array}{c}\text { QTM } \\
\text { Measurement } \\
\text { of Standard } \\
\text { Conductivity } \\
\left(\mathrm{kcal} / \mathrm{mh}^{\circ} \mathrm{C}\right)\end{array}$ & $\begin{array}{c}\text { Theoretical } \\
\text { Conductivity } \\
\text { Value of } \\
\text { Standard } \\
\left(\mathrm{kcal} / \mathrm{mh}^{\circ} \mathrm{C}\right)\end{array}$ & Date \\
\hline- & - & 20 & 4 & High & 24 & $(21+28) / 2=24.5$ & 48.5 & 0.195 & 0.196 & 8 July 1978 \\
\hline - & - & 20 & 4 & High & 24 & $(21+27) / 2=24$ & 48 & 0.196 & 0.196 & 8 July 1978 \\
\hline - & - & 20 & 4 & High & 24 & $(21+28) / 2=24.5$ & 48.5 & 0.200 & 0.196 & 8 July 1978 \\
\hline - & 一 & 20 & 4 & High & 24 & $(21+28) / 2=24.5$ & 48.5 & 0.200 & 0.196 & 8 July 1978 \\
\hline - & - & 20 & 4 & High & 24 & $(21+28) / 2=24.5$ & 48.5 & 0.200 & 0.196 & 8 July 1978 \\
\hline - & - & 20 & 4 & High & 23 & $(22+28) / 2=25$ & 48 & 0.202 & 0.196 & 9 July 1978 \\
\hline - & 一 & 20 & 4 & High & 23 & $(22+27) / 2=24.5$ & 47.5 & 0.206 & 0.196 & 9 July 1978 \\
\hline - & 一 & 20 & 4 & High & 23 & $(21+27) / 2=24.5$ & 47.5 & 0.202 & 0.196 & 9 July 1978 \\
\hline - & - & 20 & 4 & High & 23 & $(21+27) / 2=24.5$ & 47.5 & 0.201 & 0.196 & 9 July 1978 \\
\hline - & - & 20 & 4 & High & 23 & $(21+27) / 2=24.5$ & 47.5 & 0.199 & 0.196 & 9 July 1978 \\
\hline 462 & 48 & 20 & 4 & High & 24 & $24 ?$ & $48 ?$ & 0.192 & 0.196 & - \\
\hline 462 & 55 & 20 & 4 & High & 24 & $24 ?$ & $48 ?$ & 0.201 & 0.196 & - \\
\hline $462 \mathrm{~A}$ & 9 & $20 ?$ & 4 & High & $24 ?$ & $(22 ?+27) / 2=24.5$ & 48.5 & 0.194 & 0.196 & - \\
\hline $462 \mathrm{~A}$ & 32 & $20 ?$ & 4 & High & 24 & $24.5 ?$ & $48.5 ?$ & 0.196 & 0.196 & - \\
\hline $462 \mathrm{~A}$ & 38 & $20 ?$ & 4 & High & 24 & $24.5 ?$ & $48.5 ?$ & 0.203 & 0.196 & - \\
\hline $462 \mathrm{~A}$ & 40 & $20 ?$ & 4 & High & 24 & $24.5 ?$ & $48.5 ?$ & 0.202 & 0.196 & - \\
\hline $462 \mathrm{~A}$ & 51 & $20 ?$ & 4 & High & 24 & $24.5 ?$ & $48.5 ?$ & 0.199 & 0.196 & - \\
\hline $462 \mathrm{~A}$ & 64 & $20 ?$ & 4 & High & 24 & 24 & 48 & 0.200 & 0.196 & - \\
\hline $462 \mathrm{~A}$ & 65 & $20 ?$ & 4 & High & 24 & 24 & 48 & 0.203 & 0.196 & - \\
\hline $462 \mathrm{~A}$ & 67 & $20 ?$ & 4 & High & 24 & $24.5 ?$ & $48.5 ?$ & 0.200 & 0.196 & - \\
\hline $462 \mathrm{~A}$ & 68 & $20 ?$ & 4 & High & 24 & 24 & 48 & 0.201 & 0.196 & - \\
\hline $462 \mathrm{~A}$ & 74 & $20 ?$ & 4 & High & 24 & 24 & 48 & 0.200 & 0.196 & - \\
\hline
\end{tabular}

Notes: Number of samples $=22$.

Mean at $48^{\circ} \mathrm{C}=0.1996\left(\mathrm{kcal} / \mathrm{mh}^{\circ} \mathrm{C}\right)$.

Mean at $21^{\circ} \mathrm{C}=0.1861\left(\mathrm{kcal} / \mathrm{mh}^{\circ} \mathrm{C}\right)$ (temperature correction $=-0.25 \%$ per ${ }^{\circ} \mathrm{C}$ ).

Range $=+3.2 \%,-2.8 \%$.

Empirical correction factor $=K=\frac{0.1916(\mathrm{kcal}) /\left(\mathrm{mh}^{\circ} \mathrm{C}\right)}{0.1861\left(\mathrm{kcal} / \mathrm{mh}^{\circ} \mathrm{C}\right)}=1.030$.

$K=\frac{\text { true conductivity at } 21^{\circ} \mathrm{C}}{\text { measured conductivity at } 21^{\circ} \mathrm{C}}$.

It is assumed that the value of $1.16 \mathrm{kcal} / \mathrm{mh}^{\circ} \mathrm{C}$ for the QTM conductivity standard corresponds to a temperature of $30^{\circ}$ (Tabata, personal communication, 1979), and this corresponds to $1.1339 \mathrm{kcal} / \mathrm{mh}^{\circ} \mathrm{C}$ at $21^{\circ} \mathrm{C}$ (correction of $-0.25 \% \mathrm{per}{ }^{\circ} \mathrm{C}$ ).

$\left(K_{0.196}\right.$ STD $+K_{1} .16$ STD $) / 2=(1.030+1.035) / 2=1.0325=$ averaged $K$, which is used with the conductivity data measured with the QTM at these settings: time $(\mathrm{sec})=20$, heater $=4$, and mode $=$ high. The QTM measures conductivity in units of $\mathrm{kcal} / \mathrm{mh}{ }^{\circ} \mathrm{C}$. This value is $(1)$ adjusted $\left(-0.25 \%\right.$ per $\left.{ }^{\circ} \mathrm{C}\right)$ to $21^{\circ} \mathrm{C},(2)$ adjusted by using correction factor, $K$, and $(3)$ converted to units of $\left(\mathrm{cal} \bullet 10^{-3}\right) /\left(\mathrm{cm} \bullet\right.$ sec $\left.\bullet{ }^{\circ} \mathrm{C}\right)$ by using a conversion factor of 2.778 .

ards. The correction factors are (1) 1.0325 for the data determined at equipment settings of time $(s)=20$, heater $=4$, and mode $=$ high; and (2) 1.0124 for the data determined at equipment settings of time $(s)=20$, heater $=8$, and mode $=$ high .

\section{Acoustic Velocity}

Handling of sample is as discussed above, in the introduction to this appendix.

Acoustic velocity is the compressional velocity at 400 $\mathrm{kHz}$ through wet-saturated geologic material, reported in units of $\mathrm{km} / \mathrm{s}$.

Velocity was measured with a Hamilton Frame velocimeter, which is accurate to $\pm 2 \%$. The basic equipment and technique are described in Boyce (1973) and therefore will not be described here, except for calibra- tion procedures with corresponding data, and sampling techniques.

\section{Oscilloscope Calibration and Velocity-Correction Factors}

The oscilloscope used on Leg 61 was the Tektronix 485. The correction factors listed in Table 5 were used to calculate the sound-velocity data for all holes on Leg 61 . The sound-velocity measurements on the lucite, brass, and aluminum semi-standards, assuming the true velocities, are the Schreiber sound velocities listed in Table 6 (Boyce, 1973 and 1976a). In addition, distilled water, whose acoustic velocities at given temperatures are known, is also used as a standard. Apparent-velocity measurements are averaged for each semi-standard for a given $\mu \mathrm{s} / \mathrm{cm}$ setting on the oscilloscope (Tables 7-11). 
Deviations of the averages of apparent velocities measured on the velocimeter from the true velocities of the semi-standards are used to calculate a set of correction factors $(K)$ for each $\mu \mathrm{s} / \mathrm{cm}$ setting on the oscilloscope, as follows:

$$
\text { average apparent velocity }(K)=\begin{aligned}
& \text { true velocity of } \\
& \text { semi-standards }
\end{aligned}
$$

\section{Vane Shear Strength}

Vane shear strength is the shearing force $(\mathrm{kg})$ per unit area $\left(\mathrm{cm}^{2}\right)$, for water-saturated clayey sediment. For Leg 50 data, it represents primarily clayey cohesion of the material, and not (theoretically) friction of the coarser grains.

Vane shear measurements were attempted on relatively undisturbed clay samples. Vane shear strength is defined as the maximum torque applied to a vane in a clayey sample before failure. Failure theoretically occurs around the cylindrical surface area of the vane, and the final shear strength is the force $(\mathrm{kg})$ per unit area $\left(\mathrm{cm}^{2}\right)$ of the cylindrical vane. The sample is considered to be in an undrained condition. Theoretically, shear strength is made up of cohesion and friction components, and the vane shear device theoretically (not completely, in reality) measures the cohesional component of the total shear strength of the sediment.

On Leg 61, vane shear measurements were done with the DSDP Wykham Farrance Laboratory Vane Apparatus. All equipment, techniques, and calibrations are discussed in Boyce (1976b) and will not be discussed further here, except for changes from Boyce (1976a) and other pertinent information. The $1.279 \mathrm{~cm}$ (height) $\times$ $1.281 \mathrm{~cm}$ (diameter) vane (number 2) was used, and it was buried about $0.5 \mathrm{~cm}$ on top and bottom of the sample. Because it was necessary to measure the shear strength on a split core (in order to find a proper lithologic sample), the vane was inserted parallel to bedding. The torque to the spring (number 4 ) was applied at a rate of $89^{\circ} / \mathrm{min}$. The remolded test was done immediately after rotating the vane 10 times (while it is in the sample).

\section{WET-BULK DENSITY}

\section{Introduction}

During Leg 61, we used the Gamma Ray Attenuation Porosity Evaluator (GRAPE) device which was developed and described by Evans (1965) and modified as discussed by Boyce (1976a). See Boyce (1976a) for calibration, discussion, interpretation precautions, and derivation of the shore-based computer calculations of the techniques which will be discussed here.

\section{Analog GRAPE}

Calipers were not available for the Leg 61 analog GRAPE system; thus, diameters were measured by hand. All diameter adjustments to be geometric problems discussed by Boyce (1976a) have been applied. We will present only enough information here to show how, and by which equations in Boyce (1976a), the data were calculated.

The analog GRAPE data include for each core: (1) a 6.61-cm-diameter aluminum standard in liner, (2) a 2.54-cm-diameter aluminum standard in liner, and (3) the geologic core in liner. These data were processed (on the ship) through Equation 15 of Boyce (1976a) (assuming a constant attenuation coefficient of $0.1 \mathrm{~cm}^{2} / \mathrm{g}$ and a constant thickness of $6.61 \mathrm{~cm}$ for both standards and the core sample). Then, at the shore-based computer facilities, the resulting data were processed through Equation 25 (Boyce, 1976a); the corrected density of the $6.61-\mathrm{cm}$ aluminum standard $\left(\varrho_{\mathrm{ac}}\right)$ is $2.6 \mathrm{~g} / \mathrm{cm}^{3}$, and a $1.00-\mathrm{g} / \mathrm{cm}^{3}$ density is assigned to the $2.54-\mathrm{cm}$ aluminum standard. The latter is substituted for the corrected density $\left(\varrho_{\mathrm{fc}}\right)$ of the water standard. The Evans (1965) "corrected"' wet-bulk density $\left(\varrho_{b c}\right)$, obtained from Equation 25 , is then adjusted for (1) the diameter of the core (the investigator may obtain diameters from the core photographs or the DSDP repository if he wishes to manipulate the data), (2) the "corrected density" of the air, water, sediment, or breccia around the core (see Tables 12 and 13), where the diameter of the core is aligned to the gamma-ray beam (see Table 12). These geometric adjustments are applied by placing $\varrho_{b c}$ in Equations 32 through 36 of Boyce (1976a). The new, geometrically adjusted $\varrho_{b c}$ is processed through Equation 27 (Boyce, $1976 \mathrm{a})$ to adjust for the anomalous $1.128 \mathrm{-g} / \mathrm{cm}^{3}$ "corrected" density of sea water. The following parameters are used in Equation 27: grain density $\left(\varrho_{\mathrm{g}}\right)=2.7 \mathrm{~g} / \mathrm{cm}^{3}$ for sedimentary rock and $3.0 \mathrm{~g} / \mathrm{cm}^{3}$ for basalt; "corrected" grain density $\left(\varrho_{\mathrm{gc}}\right)=2.7 \mathrm{~g} / \mathrm{cm}^{3}$ for sedimentary rock and $3.0 \mathrm{~g} / \mathrm{cm}^{3}$ for basalt; fluid density $\left(\varrho_{\mathrm{f}}\right)=1.025$ $\mathrm{g} / \mathrm{cm}^{3}$; and "corrected" fluid density $\left(\varrho_{\mathrm{fc}}\right)=1.128$ $\mathrm{g} / \mathrm{cm}^{3}$. The final wet-bulk density $\left(\varrho_{\mathrm{b}}\right)$ derived from Equation 27 is the "true" wet-bulk density $( \pm 11 \%$ per each $3.17 \mathrm{~mm}$ length along the core), which assumes that all mineral grains have (1) a quartz attenuation coefficient, and (2) true and (3) corrected grain densities of $2.7 \mathrm{~g} / \mathrm{cm}^{3}$.

\section{Static 2-Minute GRAPE}

The static mode of the GRAPE allows individual samples to be counted for a 2-min period with a precision of $\pm 2 \%$. These samples are labeled as "GRAPE Special 2-Minute Count Wet-Bulk Density."

Numerous different-diameter aluminum standards were run prior to Hole 415 , and a set of $2.54-\mathrm{cm}$ and $6.61-\mathrm{cm}$ aluminum standards were processed with each data sheet. The standards were used to calculate the apparent quartz attenuation coefficient $\left(\mu_{\mathrm{a}}\right)$, using Equations 22 and 23 in Boyce (1976a). The results of the aluminum standards, which were run prior and between cores, are listed in Table 13.

The data in these tables indicate that there is a significant difference in "apparent" quartz attenuation coefficients $\left(\mu_{\mathrm{a}}\right)$ for different diameter standards, as discussed in Boyce (1976a). Therefore, we calculate our 2-minute count GRAPE data as did Boyce (1976a), in that when we use Equation 24 (Boyce, 1976a) to calcu- 


\begin{tabular}{|c|c|c|c|c|c|c|c|c|c|c|c|c|c|c|c|c|c|c|c|c|c|c|}
\hline \multirow[b]{2}{*}{ Hole } & \multirow[b]{2}{*}{ Core } & \multirow[b]{2}{*}{ Section } & \multirow[b]{2}{*}{$\begin{array}{l}\text { Interval } \\
(\mathrm{cm})\end{array}$} & \multirow{2}{*}{$\begin{array}{l}\text { Time } \\
\text { Core } \\
\text { Up } \\
\text { (hr) }\end{array}$} & \multirow{2}{*}{$\begin{array}{c}\text { Time } \\
\text { Meas. } \\
\text { (hr) }\end{array}$} & Room & $\begin{array}{l}\text { Est. } \\
\text { Core }\end{array}$ & & emp. & Temp & $\begin{array}{r}\text { Aver } \\
\text { Red a } \\
\text { Tem }\end{array}$ & $\begin{array}{l}\text { age of } \\
\text { dd Green } \\
p .\left({ }^{\circ} \mathrm{C}\right)\end{array}$ & $\begin{array}{l}\text { Est. } \\
\text { Real } \\
\text { Meas. }\end{array}$ & & TM Settir & & $\begin{array}{l}\text { Apparent } \\
\text { Condu }\end{array}$ & $\begin{array}{l}\text { Thermal } \\
\text { ctivity }\end{array}$ & $\begin{array}{l}\text { Apparent } \\
\text { Conductivity } \\
\text { Corrected to }\end{array}$ & Correction & $\begin{array}{c}\text { Trued } \mathrm{T} \\
\text { Conductivit }\end{array}$ & $\begin{array}{l}\text { hermal } \\
y^{c} \text { at } 21^{\circ} \mathrm{C}\end{array}$ \\
\hline & & & & & & $\begin{array}{l}\text { Temp. } \\
\left({ }^{\circ} \mathrm{C}\right)\end{array}$ & $\begin{array}{l}\text { Temp. } \\
\left({ }^{\circ} \mathrm{C}\right)\end{array}$ & & $\begin{array}{l}{ }^{\circ} \mathrm{Ce} \\
\left.{ }^{\circ} \mathrm{C}\right)\end{array}$ & $\begin{array}{l}\text { Green } \\
\left({ }^{\circ} \mathrm{C}\right)\end{array}$ & Actual & Estimate & $\begin{array}{l}\text { Temp, }{ }^{\text {Ta }} \\
\left({ }^{\circ} \mathrm{C}\right)\end{array}$ & $\underset{(s)}{\text { Time }}$ & Heater & Mode & $\left(\mathrm{kcal} / \mathrm{mh} \cdot{ }^{\circ} \mathrm{C}\right)$ & $\left(\frac{\mathrm{cal} \cdot 10^{-3}}{\mathrm{~cm} \cdot \sec \cdot{ }^{\circ} \mathrm{C}}\right)$ & $\left(\begin{array}{c}\left.21^{\circ} \mathrm{Cb} / \mathrm{mh} \cdot{ }^{\circ} \mathrm{C}\right)\end{array}\right.$ & $\begin{array}{l}\text { Factor, } \\
K^{2}\end{array}$ & $\left(\mathrm{kcal} / \mathrm{mh} \cdot{ }^{\circ} \mathrm{C}\right)$ & $\left(\frac{\mathrm{cal} \cdot 10^{-3}}{\mathrm{~cm} \cdot \sec \cdot{ }^{\circ} \mathrm{C}}\right)$ \\
\hline 462 & 14 & 6 & $16-26$ & $\begin{array}{l}2050 \\
0216\end{array}$ & $\begin{array}{l}0520 \\
0500\end{array}$ & 23.5 & 23.5 & 9 & (est.) & 11 & - & 10 & 33.5 & 20 & 4 & High & 1.078 & & 1.044 & 1.0325 & 1.078 & 2.998 \\
\hline $\begin{array}{l}462 \\
462\end{array}$ & $\begin{array}{l}16 \\
19\end{array}$ & $\begin{array}{l}5 \\
5\end{array}$ & $\begin{array}{l}0-10 \\
0-10\end{array}$ & $\begin{array}{l}0216 \\
0700\end{array}$ & $\begin{array}{l}0500 \\
1020\end{array}$ & & $\begin{array}{l}21.5 \\
21.5\end{array}$ & 19 & (est.) & 21 & - & 20 & 43.5 & 20 & 4 & High & 0.665 & 1.847 & 0.631 & 1.0325 & 0.651 & 1.811 \\
\hline & 19 & 5 & $\begin{array}{c}00-10 \\
120-130\end{array}$ & 0700 & $\begin{array}{l}1020 \\
1020\end{array}$ & $\begin{array}{l}23 \\
23\end{array}$ & $\begin{array}{l}21.5 \\
21.5\end{array}$ & $\begin{array}{l}23 \\
23\end{array}$ & $\begin{array}{l}\text { (est.) } \\
\text { (est.) }\end{array}$ & 25 & $\overline{-}$ & $\begin{array}{l}24 \\
24\end{array}$ & $\begin{array}{l}45.5 ? \\
45.5 ?\end{array}$ & 20 & 4 & $\begin{array}{l}\text { High } \\
\text { High }\end{array}$ & $\begin{array}{l}0.433 \\
0.994\end{array}$ & $\begin{array}{l}1.203 \\
2.761\end{array}$ & $\begin{array}{l}0.406 \\
0.933\end{array}$ & 1.0325 & $\begin{array}{l}0.419 \\
0.963\end{array}$ & \\
\hline 462 & 19 & 5 & $139-149$ & 0700 & 1020 & 23 & 21.5 & 23 & (est.) & $\begin{array}{l}25 \\
25\end{array}$ & $\bar{z}$ & $\begin{array}{l}24 \\
24\end{array}$ & $\begin{array}{l}45.5 ? \\
45.5 ?\end{array}$ & $\begin{array}{l}20 \\
20\end{array}$ & $\begin{array}{l}4 \\
4\end{array}$ & $\begin{array}{l}\text { High } \\
\text { High }\end{array}$ & $\begin{array}{l}0.994 \\
1.045\end{array}$ & $\begin{array}{l}2.761 \\
2.903\end{array}$ & $\begin{array}{l}0.933 \\
0.981\end{array}$ & $\begin{array}{l}1.0325 \\
1.0325\end{array}$ & $\begin{array}{l}0.963 \\
1.013\end{array}$ & $\begin{array}{l}2.678 \\
2.816\end{array}$ \\
\hline 462 & 24 & 5 & $0-10$ & 1835 & 2030 & 23.5 & 19.5 & 9 & $\begin{array}{l}\text { (est.) } \\
\text { (e) }\end{array}$ & II & - & 10 & 29.5 & 20 & 4 & $\mathrm{High}$ & 0.990 & 2.750 & 0.969 & 1.0325 & 1.000 & 2.781 \\
\hline 462 & 28 & 6 & 44-54 & 0255 & 0645 & 24 & 23 & 10 & (est.) & 12.5 & - & 11 & 34 & 20 & 4 & High & 1.089 & 3.294 & 1.054 & 1.0325 & 1.088 & 3.024 \\
\hline 462 & 28 & 6 & $139-149$ & 0255 & 0645 & 24 & 23 & 9 & (est.) & 11 & - & 10 & 33 & 20 & 4 & High & 1.027 & 2.853 & 0.996 & 1.0325 & 1.029 & 2.859 \\
\hline 462 & 37 & 1 & $20-30$ & 2215 & 0210 & 24 & 23 & 12 & (est.) & 14 & - & 13 & 36 & 20 & 4 & High & 1.006 & 2.797 & 0.968 & 1.0325 & 0.999 & 2.780 \\
\hline 462 & 39 & 1 & $1-11$ & 0133 & 0730 & 24.5 & 23 & 9. & (est.) & 11 & - & 10 & 34 & 20 & 4 & High & 1.124 & 3.122 & 1.087 & 1.0325 & 1.122 & 3.121 \\
\hline $\begin{array}{l}462 \\
462\end{array}$ & 39 & 1 & $\begin{array}{l}48-58 \\
25-35\end{array}$ & 0133 & 0730 & 24.5 & 24 & 11.5 & 5 (est.) & 13.5 & - & 12.5 & 36 & 20 & 4 & High & 0.837 & 2.325 & 0.806 & 1.0325 & 0.832 & 2.312 \\
\hline $\begin{array}{l}402 \\
462\end{array}$ & $\begin{array}{l}48 \\
49\end{array}$ & $\begin{array}{l}1 \\
2\end{array}$ & $\begin{array}{c}255-35 \\
117-128\end{array}$ & $\begin{array}{l}1918 \\
2100\end{array}$ & 0900 & 24 & ${ }_{233}^{23}$ & 11 & (est.) & 13 & - & 12 & 35 & 20 & 4 & High & 1.434 & 3.984 & 1.384 & 1.0325 & 1.428 & $\begin{array}{l}3.971 \\
3.182\end{array}$ \\
\hline 462 & 49 & 3 & $53-61$ & 2100 & $?$ & $\begin{array}{l}24 \\
24\end{array}$ & $\begin{array}{l}233 ? \\
\text { ? }\end{array}$ & 10 & (est.) & $\begin{array}{l}11 \\
12\end{array}$ & $\bar{z}$ & $\begin{array}{l}10 \\
11\end{array}$ & $\begin{array}{l}33 ? \\
34 ?\end{array}$ & 20 & $\begin{array}{l}4 \\
4\end{array}$ & $\begin{array}{l}\text { High } \\
\text { High }\end{array}$ & $\begin{array}{l}1.120 \\
1.419\end{array}$ & $\begin{array}{l}3.1111 \\
3.942\end{array}$ & $\begin{array}{l}1.086 ? \\
1.373\end{array}$ & $\begin{array}{l}1.0325 \\
100325\end{array}$ & $\begin{array}{l}1.121 ? ? \\
4418\end{array}$ & $\begin{array}{l}3.118 ? \\
3.940\end{array}$ \\
\hline 462 & 50 & 5 & $140-150$ & 2255 & 0437 & 24 & 23.5 & 10 & (est.) & 12 & 11 & 11 & 34.5 & 20 & 4 & $\begin{array}{l}\text { Mign } \\
\text { High }\end{array}$ & 1.099 & $\begin{array}{l}3.9422 \\
3.053\end{array}$ & 1.062 & 1.0325 & 1.096 & $\begin{array}{l}3.9400 \\
3.047\end{array}$ \\
\hline 462 & 50 & 4 & $1-11$ & 2255 & 0437 & 24 & 23.5 & 9.5 & 5 (est.) & 11.5 & 10.5 & & 34 & 20 & 4 & High & 1.003 & 2.786 & 0.970 & 1.0325 & 1.002 & 2.786 \\
\hline 462 & 50 & 2 & $128-138$ & 2255 & 0437 & 24 & 23.5 & 13 & (est.) & is & 14 & - & 37.5 & 20 & 4 & High & 1.017 & 2.825 & 0.975 & 1.0325 & 1.007 & 2.798 \\
\hline 462 & 51 & 1 & $95-105$ & 0025 & & & 23.5 & 9 & (est.) & II & - & 10 & 33.5 & 20 & 4 & High & 1.526 & 4.239 & 1.478 & 1.0325 & 1.526 & 4.243 \\
\hline 462 & 51 & 2 & $60-70$ & 0025 & 0700 & 24 & 23.5 & 11 & (est.) & 13 & - & 12 & 35.5 & 20 & 4 & High & 1.071 & 2.975 & 1.032 & 1.0325 & 1.066 & $\begin{array}{l}2.962 \\
4.403\end{array}$ \\
\hline 462 & 51 & 2 & $95-105$ & 0025 & 0700 & 24 & 23.5 & 8.5 & 5 (est.) & 10.5 & - & 9.5 & 33 & 20 & 4 & High & 1.581 & 4.392 & 1.534 & 1.0325 & 1.584 & $\begin{array}{l}4.403 \\
3.301\end{array}$ \\
\hline 462 & 52 & 1 & $0-10$ & 0234 & 0845 & 24 & 23.5 & 9 & (est.) & 10 & - & 9 & 32.5 & 20 & 4 & High & 1.184 & 3.289 & 1.150 & 1.0325 & 1.187 & $\begin{array}{l}3.301 \\
5.116\end{array}$ \\
\hline 462 & 54 & 1 & $3-13$ & 1230 & 1502 & 24 & 21.5 & 14 & (est.) & 16 & - & 15 & 36.5 & 20 & 8 & High & 1.891 & 5.253 & 1.818 & 1.0124 & 1.840 & $\begin{array}{l}5.116 \\
4.107\end{array}$ \\
\hline 462 & 54 & 3 & 78-88 & $\overline{-1}$ & 1502 & 24 & 21.5 & 14 & (est.) & 16 & - & 15 & 36.5 & 20 & 8 & High & 1.518 & 4.217 & 1.459 & 1.0124 & 1.478 & $\begin{array}{l}4.107 \\
4.417\end{array}$ \\
\hline 462 & 55 & 1 & $80-90$ & 1445 & 1700 & 24 & 21 & 16 & (est.) & 18 & - & 17 & 38 & 20 & 8 & High & 1.639 & 4.553 & 1.569 & 1.0124 & 1.589 & $\begin{array}{l}4.417 \\
4.247\end{array}$ \\
\hline 462 & 55 & 3 & $50-60$ & 1445 & 1700 & 24 & 21 & 15 & & 17 & - & 16 & 37 & 20 & 8 & High & 1.572 & 4.367 & 1.509 & 1.0124 & 1.527 & $\begin{array}{l}4.247 \\
2.470 ?\end{array}$ \\
\hline $\begin{array}{l}462 \\
462\end{array}$ & 56 & $\frac{1}{3}$ & $\begin{array}{l}95-100 \\
45-47\end{array}$ & 1645 & $?$ & $\begin{array}{l}24 \\
24\end{array}$ & $\begin{array}{l}21 ? ? \\
21 ?\end{array}$ & 17 & $\begin{array}{l}\text { (est.) } \\
\text { (esti) }\end{array}$ & 19 & - & 18 & $39 ?$ & 20 & 4 & $\begin{array}{l}\text { High } \\
\text { Higr }\end{array}$ & $\begin{array}{l}0.901 \\
1070\end{array}$ & 2.503 & 0.860 & 1.0325 & $0.889 ?$ & $\begin{array}{l}2.470 ? \\
2.987\end{array}$ \\
\hline $\begin{array}{l}402 \\
462\end{array}$ & 58 & 1 & $\begin{array}{l}45-4 ! \\
65-75\end{array}$ & $\begin{array}{l}1900 \\
2100\end{array}$ & 0100 & $\begin{array}{l}24 \\
24\end{array}$ & ${ }_{23}^{21 ?}$ & $\begin{array}{l}10 \\
10.5\end{array}$ & 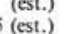 & $\begin{array}{l}12 \\
12.5\end{array}$ & $\bar{z}$ & $\begin{array}{l}11 \\
11.5\end{array}$ & $\begin{array}{l}32 ? \\
34.5\end{array}$ & $\begin{array}{l}20 \\
20\end{array}$ & $\begin{array}{l}4 \\
4\end{array}$ & $\begin{array}{l}\text { High } \\
\text { High }\end{array}$ & $\begin{array}{l}1.070 \\
0.974\end{array}$ & $\begin{array}{r}2.972 \\
2.706\end{array}$ & $\begin{array}{l}1.041 \\
0.941\end{array}$ & $\begin{array}{l}1.0325 \\
1.0325\end{array}$ & $\begin{array}{l}1.074 \\
0.9772\end{array}$ & $\begin{array}{l}2.987 \\
2.701\end{array}$ \\
\hline 462 & 58 & 4 & $71-78$ & 2100 & 0100 & 24 & 23 & 10.5 & & 12.5 & $\bar{z}$ & 11.5 & 34.5 & 20 & 4 & High & 1.168 & 3.245 & 1.129 & 1.0325 & 1.165 & 3.239 \\
\hline 462 & 58 & 4 & $128-138$ & 2100 & 0100 & 24 & 23 & 11 & (est.) & 13 & - & 12 & 35 & 20 & 4 & High & 1.402 & 3.895 & 1.353 & 1.0325 & 1.396 & 3.883 \\
\hline 462 & 59 & i & $43-53$ & 2315 & 0230 & 24 & 23 & 12.5 & 5 (est.) & 14.5 & - & 13.5 & 36.5 & 20 & 4 & High & 1.090 & 3.028 & 1.048 & 1.0325 & 1.081 & 3.007 \\
\hline 462 & 59 & $i$ & $60-70$ & 2315 & 0230 & 24 & 23 & 10.5 & & 12.5 & $=$ & 11.5 & 31.5 & 20 & 4 & High & 1.045 & 2.903 & 1.018 & 1.0325 & 1.051 & 2.920 \\
\hline 462 & 60 & 2 & $55-65$ & 0425 & 0835 & 24 & 23 & 10.5 & 5 (est.) & 12.5 & - & 11.5 & 31.5 & 20 & 4 & High & 1.356 & 3.767 & 1.320 & 1.0325 & 1,363 & $\begin{array}{l}3.790 \\
4.774\end{array}$ \\
\hline 462 & 61 & i & $50-60$ & 1127 & 1300 & 24 & 19.2 & 16 & (est.) & 18 & - & 17 & 36.2 & 20 & 8 & High & 1.763 & 4.898 & 1.696 & 1.0124 & 1.717 & $\begin{array}{l}4.774 \\
6097\end{array}$ \\
\hline 462 & 62 & $i$ & $80-90$ & 1550 & 1700 & 24 & 18.6 & 17 & (est.) & 19 & - & 18 & 36.6 & 20 & 8 & High & 2.587 & 7.187 & 2.486 & 1. 0124 & 2.517 & 6.997 \\
\hline 462 & 62 & 2 & $107-117$ & 1550 & 1700 & 24 & 18.6 & 18 & (est.) & 20 & - & 19 & 37.6 & 20 & 8 & High & 1.602 & 4.450 & 1.536 & 1.0124 & 1.555 & 4.321 \\
\hline 462 & 63 & 2 & $32-34$ & 2046 & 2215 & 24 & 19 & 17 & (est.) & 19 & - & 18 & 37 & 20 & 8 & High & 1.536 & 4.267 & 1.475 & 1.0124 & 1.493 & $\begin{array}{l}4.150 \\
3.491\end{array}$ \\
\hline 462 & 63 & 3 & $117-120$ & 2046 & 2215 & 24 & 19 & 17 & (est.) & 19 & - & 18 & 37 & 20 & 8 & High & 1.292 & 3.589 & 1.240 & 1.0124 & 1.256 & 3.491 \\
\hline 462 & 64 & 3 & $45-55$ & 0053 & 0430 & 24 & 23 & 8.5 & 5 (est.) & 10.5 & - & 9.5 & 32.5 & 20 & 4 & High & 1.498 & 4.16 & 1.455 & 1.0325 & 1.502 & $\begin{array}{l}4.176 \\
7.265\end{array}$ \\
\hline 462 & 65 & 1 & $34-44$ & 0400 & 0645 & 24 & 23 & 16 & (est.) & 18 & - & 17 & 40 & 20 & 8 & High & 2,710 & 7.528 & 2.581 & 1.0124 & 2.613 & $\begin{array}{l}7.265 \\
4.671\end{array}$ \\
\hline 462 & 65 & 2 & 6-16 & 0400 & $0645 ?$ & 24 & 23 & $\begin{array}{l}20 \\
18\end{array}$ & (est.) & 22 & - & 21 & 44 & 20 & 8 & High & 1.761 & 4.892 & 1.660 & 1.0124 & 1.680 & $\begin{array}{l}4.671 \\
4,793\end{array}$ \\
\hline $\begin{array}{l}462 \\
462\end{array}$ & $\begin{array}{l}66 \\
66\end{array}$ & $\frac{1}{2}$ & $\begin{array}{l}20-30 \\
56-70\end{array}$ & 0942 & 1040 & 24 & 18 & 18 & $\begin{array}{l}\text { (est.) } \\
\text { (est) }\end{array}$ & 20 & - & 19 & 37 & 20 & 8 & $\begin{array}{l}\text { High } \\
\text { Hiag }\end{array}$ & 1.774 & 4.928 & 1.703 & 1.0124 & 1.724 & $\begin{array}{r}4.793 \\
5.098\end{array}$ \\
\hline $\begin{array}{l}402 \\
462\end{array}$ & $\begin{array}{l}66 \\
66\end{array}$ & $\begin{array}{l}2 \\
4\end{array}$ & 列 & $\begin{array}{l}0942 \\
0942\end{array}$ & $\begin{array}{l}1100 \\
1140\end{array}$ & $\begin{array}{l}24 \\
24\end{array}$ & $\begin{array}{l}19 \\
20.5\end{array}$ & $\begin{array}{l}16 \\
16\end{array}$ & $\begin{array}{l}\text { (est.) } \\
\text { (est) }\end{array}$ & 18 & - & 17 & $\begin{array}{l}36 \\
475\end{array}$ & 20 & 8 & $\begin{array}{l}\text { High } \\
\text { Hiagh }\end{array}$ & $\begin{array}{l}1,882 \\
789\end{array}$ & 5.228 & 1.811 & 1.0124 & 1.834 & $\begin{array}{l}5.098 \\
4.701\end{array}$ \\
\hline $\begin{array}{l}402 \\
462\end{array}$ & 66 & 5 & $50-60$ & 0942 & 1700 & 24 & 21.3 & $\begin{array}{l}16 \\
17\end{array}$ & 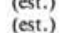 & 19 & $\bar{z}$ & 18 & $\begin{array}{l}49.3 \\
39.3\end{array}$ & $\begin{array}{l}20 \\
20\end{array}$ & $\begin{array}{l}8 \\
8\end{array}$ & $\begin{array}{l}\mathrm{High} \\
\mathrm{High}\end{array}$ & 1.78 & $\begin{array}{l}\begin{array}{r}4.970 \\
4.786\end{array} \\
4\end{array}$ & $\begin{array}{l}1.6 \\
1.6\end{array}$ & $\begin{array}{l}1.0124 \\
1.124\end{array}$ & $\begin{array}{l}1.692 \\
1.664\end{array}$ & $\begin{array}{l}4.6101 \\
4.627\end{array}$ \\
\hline 462 & 66 & 6 & $2-12$ & 0942 & 1210 & 24 & 21.5 & 18 & (est.) & 20 & - & 19 & 40.5 & 20 & 8 & $\mathrm{High}$ & 1.95 & 5.420 & 1.8 & 1.0124 & 1.879 & 5.223 \\
\hline 462 & 67 & i & $7-19$ & 1410 & 1500 & 24 & 17.6 & 17 & (est.) & 19 & - & 18 & 35.6 & 20 & 8 & $\mathrm{High}$ & 1.66 & 4.631 & $\begin{array}{l}1.606 \\
1.006\end{array}$ & 1.0124 & 1.626 & 4.521 \\
\hline 462 & 67 & 2 & $125-135$ & 1410 & 1530 & 24 & 18.6 & 20 & (est.) & 22 & - & 21 & 39.6 & 20 & 8 & High & 1.900 & 5.278 & 1.812 & 1.0124 & 1.834 & 5.099 \\
\hline 462 & 67 & $i$ & $7-19$ & 1410 & 1600 & 24 & 20.2 & 14 & (est.) & 16 & - & 15 & 35.2 & 20 & 8 & High & 1.518 & 4.217 & 1.464 & 1.0124 & 1.482 & 4.120 \\
\hline 462 & 67 & 2 & $125-135$ & 1410 & 1630 & 24 & 21.3 & 14 & (est.) & 16 & - & 15 & 36.3 & 20 & 8 & High & 1.993 & 5.537 & 1.917 & 1.0124 & 1.941 & 5.394 \\
\hline 462 & 68 & 2 & $62-72$ & 2010 & 2145 & 24 & 19.5 & 16 & $\begin{array}{l}\text { (est.) } \\
\text { (a) }\end{array}$ & 18 & - & 17 & 36.5 & 20 & 8 & $\mathrm{High}$ & 1.585 & 4.403 & 1.524 & 1.0124 & 1.542 & 4.288 \\
\hline 462 & 68 & 3 & $11-20$ & 2010 & 2200 & 24 & 20.2 & 16 & (est.) & 18 & - & 17 & 37.2 & 20 & 8 & High & 1.470 & 4.084 & 1.410 & 1.0124 & 1.428 & 3.969 \\
\hline 462 & 68 & 3 & $98-118$ & 2010 & 2215 & 24 & 20.8 & 15 & (est.) & 17 & - & 16 & 36.8 & 20 & 8 & High & 1.870 & 5.1 & 1.796 & 1.0124 & 1.819 & 5.055 \\
\hline 462 & 69 & 1 & $20-30$ & 0005 & 0115 & 23 & 18.6 & 13.5 & 5 (est.) & 15.5 & - & 14.5 & 33 & 20 & 8 & High & 2.5 & 6.987 & 2.4 & 1.0124 & 2.469 & 6.864 \\
\hline 462 & 69 & 2 & $34-44$ & 0005 & 0115 & 23 & 18.6 & 14 & (est.) & 16 & - & is & 33 & 20 & 8 & High & 1.9 & 5.4 & 1.8 & 1.0 & 1.909 & 5,307 \\
\hline $462 \mathrm{~A}$ & 7 & 1 & $38-50$ & 1255 & 1515 & 24 & 21.4 & 17 & (est.) & $19 t$ & - & 18 & 39 & 20 & 4 & High & 1.101 & 3.0 & 1.0 & 1.0325 & 1.084 & 3.013 \\
\hline $462 \mathrm{~A}$ & $\mathrm{H}-3$ & 2 & 6-14 & 1600 & 1800 & 24 & 20.6 & 17 & (est.) & & - & 18 & 38 & 20 & 4 & High & 1.899 & 5.2 & 1.8 & 1.0325 & 1.874 & 5.210 \\
\hline $462 \mathrm{~A}$ & $\mathrm{H}-4$ & 2 & $25-35$ & 0135 & & 24 & 21 & 19 & (est.) & 211 & - & 20 & $41 ?$ & 20 & 8 & High & 1.901 & 5.2 & 1.8 & 1.0124 & 1.828 & 5.082 \\
\hline $462 \mathrm{~A}$ & $\mathrm{H}-4$ & 2 & $107-117$ & 0135 & 0340 & & 21 & 17 & (est.) & 19 ( & - & 13.5 & 39.5 & 20 & 8 & High & 2.018 & 5.606 & 1.925 & 1.0124 & 1.949 & 5.417 \\
\hline $462 \mathrm{~A}$ & $\mathrm{H}-4$ & 3 & $38-48$ & 0135 & & 24 & 21 & 9 & (est.) & 11 (6 & - & 10 & 31 & 20 & 4 & High & 1.620 & 4.500 & 1.580 & 1.0325 & 1.631 & $\begin{array}{r}4.535 \\
4626\end{array}$ \\
\hline${ }_{462 \mathrm{~A}}^{462 \mathrm{~A}}$ & $\begin{array}{l}\mathrm{H}-4 \\
8\end{array}$ & 3 & $64-74$ & 0135 & 0340 & 24 & 21 & 9 & (est.) & 11 (est.) & - & 10 & 31 & 20 & 4 & $\mathrm{Hi}_{\mathrm{H}}$ & 1.653 & 4.592 & 1.6 & 1.0325 & 1.664 & $\begin{array}{l}4.626 \\
3.921\end{array}$ \\
\hline 462A & $\begin{array}{l}8 \\
9\end{array}$ & 3 & $\begin{array}{l}131-141 \\
125-137\end{array}$ & $\begin{array}{l}1825 \\
0550\end{array}$ & ? & $\begin{array}{l}24 \text { (estimate) } \\
24 \text { ? }\end{array}$ & $20 ?$ & $\begin{array}{l}16 \\
18\end{array}$ & $\begin{array}{l}\text { (est.) } \\
\text { (set) }\end{array}$ & 18 (est.) & $\bar{z}$ & 17 & $\begin{array}{l}37 ? ? \\
40\end{array}$ & 20 & 8 & $\begin{array}{l}\text { High } \\
\text { Hiagh }\end{array}$ & 1.451 & 4.031 & 1.393 & 1.0124 & 1.410 & $\begin{array}{l}3.921 \\
4.087\end{array}$ \\
\hline $\begin{array}{l}462 \mathrm{~A} \\
462 \mathrm{~A}\end{array}$ & $\begin{array}{l}9 \\
9\end{array}$ & $\begin{array}{l}1 \\
4\end{array}$ & $\begin{array}{c}125-137 \\
12-21\end{array}$ & $\begin{array}{l}0550 \\
0550\end{array}$ & ? & $\begin{array}{l}24 ? \\
24 ?\end{array}$ & ${ }_{21}^{21}$ & $\begin{array}{l}18 \\
19\end{array}$ & $\begin{array}{l}\text { (est.) } \\
\text { (est) }\end{array}$ & 20 (est.) & $\bar{z}$ & ${ }_{20}^{19}$ & $\begin{array}{l}40 \\
41\end{array}$ & 20 & $\begin{array}{l}8 \\
8\end{array}$ & $\begin{array}{l}\text { High } \\
\text { High }\end{array}$ & 1.524 & $\begin{array}{l}4.234 \\
3.953\end{array}$ & 1.452 & 1.0124 & 1.470 & $\begin{array}{l}4.087 \\
3.806\end{array}$ \\
\hline $462 \mathrm{~A}$ & 17 & i & $86-95$ & 0810 & 0900 & $\begin{array}{l}24 ? \\
24 ?\end{array}$ & $\begin{array}{l}21.5 \\
17.5\end{array}$ & 17 & $\begin{array}{l}\text { (est.) } \\
\text { (est.) }\end{array}$ & $\begin{array}{l}21 \text { (est.) } \\
19 \text { (est.) }\end{array}$ & $=$ & $\begin{array}{l}20 \\
18\end{array}$ & $\begin{array}{l}41 \\
35.5\end{array}$ & $\begin{array}{l}20 \\
20\end{array}$ & $\begin{array}{l}8 \\
8\end{array}$ & $\begin{array}{l}\text { High } \\
\text { High }\end{array}$ & $\begin{array}{l}1.423 \\
1.728\end{array}$ & $\begin{array}{l}3.993 \\
4.800\end{array}$ & $\begin{array}{l}1.352 \\
1.665\end{array}$ & $\begin{array}{l}1.0124 \\
1.0124\end{array}$ & $\begin{array}{l}\begin{array}{l}1.369 \\
1.686\end{array} \\
\end{array}$ & $\begin{array}{l}3.806 \\
4.687\end{array}$ \\
\hline $462 \mathrm{~A}$ & 17 & 2 & $48-58$ & 0810 & 0900 & 24 ? & 17.5 & 16 & (est.) & 18 (est.) & $=$ & 17 & 34.5 & 20 & 8 & High & 1.896 & 5.267 & 1.832 & 1.0124 & 1.855 & 5.156 \\
\hline $462 \mathrm{~A}$ & 18 & 2 & $11-21$ & 1525 & ? & $24 ?$ & $20 ?$ & & - & - & 18 & - & $38 ?$ & 20 & 8 & High & 1.667 & 4.631 & $1.596 ?$ & 1.0124 & 1.615? & $4.490 ?$ \\
\hline
\end{tabular}




\begin{tabular}{|c|c|c|c|c|c|c|c|c|c|c|c|c|c|c|c|c|c|c|c|c|c|}
\hline $462 \mathrm{~A}$ & 20 & 1 & 23-32 & 1645 & - & 24 & 24 & - & - & 19.5 & - & 43.5 & 20 & 8 & High & 1.614 & 4.483 & 1.523 & 1.0124 & 1.542 & 4.287 \\
\hline $462 \mathrm{~A}$ & 20 & 2 & $2-12$ & 1645 & $\overline{x m}$ & 24 & 24 & - & - & 18 & - & 42 & 20 & 8 & High & 1.698 & 7.717 & 1.609 & 1.0124 & 1.629 & 4.528 \\
\hline $462 \mathrm{~A}$ & 24 & 1 & $12-22$ & 1420 & 1600 & 24 & 19.6 (est.) & $\bar{z}$ & $\bar{z}$ & $\begin{array}{l}24 \\
10\end{array}$ & - & $\begin{array}{l}43.6 \\
38.6\end{array}$ & 20 & 8 & High & 1.569 & $\begin{array}{l}4.359 \\
\end{array}$ & 1.480 & 1.0124 & 1.498 & $\begin{array}{l}4.165 \\
3855\end{array}$ \\
\hline $462 \mathrm{~A}$ & 24 & 2 & 6-15 & $\begin{array}{l}1420 \\
0825\end{array}$ & 1600 & 24 & 19.6 (est.) & $\bar{z}$ & $\bar{z}$ & $\begin{array}{l}19 \\
19\end{array}$ & - & 38.6 & 20 & 8 & $\begin{array}{l}\text { High } \\
\text { High }\end{array}$ & 1.433 & 3.984 & 1.370 & 1.0124 & 1.387 & $\begin{array}{l}3.855 \\
4360\end{array}$ \\
\hline $462 \mathrm{~A}$ & 28 & $\frac{1}{2}$ & $\begin{array}{l}115-125 \\
88-95\end{array}$ & $\begin{array}{l}0825 \\
0825\end{array}$ & 0930 & ${ }_{24}^{24}$ & & $\bar{z}$ & $\bar{z}$ & $\begin{array}{l}19 \\
19\end{array}$ & $\bar{z}$ & $\begin{array}{l}37 \\
38\end{array}$ & $\begin{array}{l}20 \\
20\end{array}$ & $\begin{array}{l}8 \\
8\end{array}$ & $\begin{array}{l}\text { High } \\
\text { High }\end{array}$ & $\begin{array}{l}1.610 \\
1.763\end{array}$ & $\begin{array}{r}4.473 \\
4898\end{array}$ & $\begin{array}{l}1.546 \\
1.688\end{array}$ & $\begin{array}{l}1.0124 \\
10124\end{array}$ & 1.565 & $\begin{array}{l}4.352 \\
4.751\end{array}$ \\
\hline $\begin{array}{l}462 \mathrm{~A} \\
462 \mathrm{~A}\end{array}$ & $\begin{array}{l}28 \\
28\end{array}$ & 2 & $\begin{array}{l}85-95 \\
71-81\end{array}$ & $\begin{array}{l}0825 \\
0825\end{array}$ & $\begin{array}{l}0950 \\
0950\end{array}$ & 24 & $\begin{array}{l}19 \text { (est.) } \\
19 \text { (est.) }\end{array}$ & $\overline{-}$ & $\bar{z}$ & $\begin{array}{l}19 \\
18\end{array}$ & $\overline{-}$ & $\begin{array}{l}38 \\
37\end{array}$ & $\begin{array}{l}20 \\
20\end{array}$ & $\begin{array}{l}8 \\
8\end{array}$ & $\begin{array}{l}\text { High } \\
\text { High }\end{array}$ & $\begin{array}{l}1.763 \\
1.669\end{array}$ & $\begin{array}{l}4.898 \\
4.636\end{array}$ & 1.688 & $\begin{array}{l}1.0124 \\
1.024\end{array}$ & $\begin{array}{l}1.709 \\
1.07\end{array}$ & $\begin{array}{l}4.751 \\
4.510\end{array}$ \\
\hline $\begin{array}{l}462 \mathrm{~A} \\
462 \mathrm{~A}\end{array}$ & $\begin{array}{l}28 \\
28\end{array}$ & 4 & $\begin{array}{c}13-81 \\
135-145\end{array}$ & $\begin{array}{l}0825 \\
0825\end{array}$ & $\begin{array}{l}9950 \\
0950\end{array}$ & $\begin{array}{l}24 \\
24\end{array}$ & 19 (est.) & $\bar{z}$ & $\bar{z}$ & $\begin{array}{l}18 \\
20\end{array}$ & $\bar{z}$ & $\begin{array}{l}37 \\
39\end{array}$ & $\begin{array}{l}20 \\
20\end{array}$ & $\begin{array}{l}8 \\
8\end{array}$ & $\begin{array}{l}\text { High } \\
\text { High }\end{array}$ & $\begin{array}{l}1.669 \\
1.710\end{array}$ & $\begin{array}{l}4.636 \\
4.750\end{array}$ & $\begin{array}{l}1.602 \\
.633\end{array}$ & $\begin{array}{l}1.0124 \\
10124\end{array}$ & $\begin{array}{l}1.622 \\
1.653\end{array}$ & $\begin{array}{l}4.510 \\
4.596\end{array}$ \\
\hline $462 \mathrm{~A}$ & 29 & 2 & $113-123$ & 1750 & 0950 & 24 & 17.5 & - & - & 18 & $=$ & 35.5 & 20 & 8 & High & 1.786 & $\begin{array}{l}4.750 \\
4.962\end{array}$ & $\begin{array}{l}1.633 \\
1.721\end{array}$ & $\begin{array}{l}1.00124 \\
1.0124\end{array}$ & $\begin{array}{l}1.633 \\
1.742\end{array}$ & $\begin{array}{l}4.596 \\
4.844\end{array}$ \\
\hline $462 \mathrm{~A}$ & 29 & 4 & $66-76$ & 1750 & 2010 & 24 & 19 & - & - & 20 & - & 39 & 20 & 8 & High & 1.896 & 5.267 & 1.811 & 1.0124 & 1.833 & 5.096 \\
\hline $462 \mathrm{~A}$ & 30 & 1 & $38-48$ & 0315 & 0430 & 23 & 19 & - & - & 18 & - & 36 & 20 & 8 & High & 1.786 & 4.962 & 1.719 & 1.0124 & 1.740 & 4.838 \\
\hline $462 \mathrm{~A}$ & 30 & 2 & $30-40$ & 0315 & 0430 & 23 & 19 & - & - & 16.5 & - & 34.5 & 20 & 8 & High & 1.979 & 5.498 & 1.912 & 1.0124 & 1.936 & 5.381 \\
\hline $462 \mathrm{~A}$ & 30 & 3 & $16-26$ & 0315 & 0430 & 23 & 19 & - & - & 18.5 & - & 36.5 & 20 & 8 & High & 1.469 & 4.081 & 1.412 & 1.0124 & 1.430 & 3.974 \\
\hline $462 \mathrm{~A}$ & 30 & 4 & $7-17$ & 0315 & 0430 & 23 & 19 & - & - & 17.5 & - & 35.5 & 20 & 8 & High & 1.910 & 5,306 & 1.841 & 1.0124 & 1.864 & 5.181 \\
\hline $462 \mathrm{~A}$ & 32 & 2 & $42-45$ & 1000 & 1115 & 24 & 19 & - & - & 21 & - & 40 & 20 & 8 & High & 1.243 & 3.453 & 1.184 & 1.0124 & 1.199 & 3.332 \\
\hline $462 \mathrm{~A}$ & 38 & 1 & $89-91$ & 2113 & 2230 & 24 & 19 & - & - & 19 & - & 38 & 20 & 8 & High & 1.618 & 4.495 & 1.549 & 1.0124 & 1.568 & 4.360 \\
\hline $462 \mathrm{~A}$ & 38 & 2 & 6-8 & 2113 & 2230 & 24 & 19 & - & - & 18 & - & 37 & 20 & 8 & High & 1.761 & 4.892 & 1.691 & 1.0124 & 1.711 & 4.758 \\
\hline $462 \mathrm{~A}$ & 39 & 1 & $137-147$ & 0450 & 0600 & $24 ?$ & 19.5 & - & - & 18 & - & 37.5 & 20 & 8 & High & 1.339 & 3.720 & 1.284 & 1.0124 & 1.300 & 3.614 \\
\hline $462 \mathrm{~A}$ & 39 & 2 & 101-111 & 0450 & 0600 & $24 ?$ & 19.5 & - & - & 19 & - & 38.5 & 20 & 8 & High & $2.144 ?$ & 5.956 & $2.050 ?$ & 1.0124 & 2.076 & 5.770 \\
\hline $462 \mathrm{~A}$ & 39 & 3 & $115-125$ & 0450 & 0600 & $24 ?$ & 19.5 & - & - & 18 & - & 37.5 & 20 & 8 & High & 1.683 & 4.675 & 1.614 & 1.0124 & 1.634 & $\begin{array}{l}4.541 \\
3.432\end{array}$ \\
\hline $462 \mathrm{~A}$ & 40 & 1 & $95-105$ & $\begin{array}{l}0830 \\
0\end{array}$ & 1000 & 24 & 19.2 & $\bar{z}$ & - & ${ }_{20}^{21}$ & - & $\begin{array}{l}40.2 \\
39.2\end{array}$ & 20 & 8 & High & 1.281 & 3.559 & 1.220 & 1.0124 & 1.234 & $\begin{array}{l}3.432 \\
4.60\end{array}$ \\
\hline $462 \mathrm{~A}$ & $\begin{array}{l}40 \\
45\end{array}$ & 2 & $\begin{array}{l}42-44 \\
10-110\end{array}$ & 0830 & 1000 & 24 & $\begin{array}{l}19.2 \\
19.2\end{array}$ & $=$ & $\bar{z}$ & 20 & $\bar{z}$ & $\begin{array}{l}39.2 \\
39.7\end{array}$ & ${ }_{20}^{20}$ & $\begin{array}{l}8 \\
8\end{array}$ & $\begin{array}{l}\text { High } \\
\text { High }\end{array}$ & $\begin{array}{l}1.623 \\
1.657\end{array}$ & $\begin{array}{r}4.509 \\
4.603\end{array}$ & $\begin{array}{l}1.549 \\
1580\end{array}$ & 1.0124 & $\begin{array}{l}1.568 \\
599\end{array}$ & $\begin{array}{l}4.360 \\
\end{array}$ \\
\hline $\begin{array}{l}462 \mathrm{~A} \\
462 \mathrm{~A}\end{array}$ & $\begin{array}{l}45 \\
45\end{array}$ & $\begin{array}{l}1 \\
3\end{array}$ & $\begin{array}{c}100-110 \\
1-11\end{array}$ & $\begin{array}{l}0300 \\
0300\end{array}$ & $\begin{array}{l}0430 \\
0430\end{array}$ & $\begin{array}{l}23 \\
23\end{array}$ & $\begin{array}{l}19.2 \\
19.2\end{array}$ & $\overline{-}$ & $=$ & $\begin{array}{l}20.5 \\
19\end{array}$ & $=$ & $\begin{array}{l}39.7 \\
338.2\end{array}$ & 20 & $\begin{array}{l}8 \\
8\end{array}$ & $\begin{array}{l}\text { High } \\
\text { High }\end{array}$ & $\begin{array}{l}1.657 \\
1.679\end{array}$ & $\begin{array}{l}4.603 \\
4.664\end{array}$ & $\begin{array}{l}1.580 \\
1.607\end{array}$ & $\begin{array}{l}1.0124 \\
10.24\end{array}$ & $\begin{array}{r}1.599 \\
.627\end{array}$ & $\begin{array}{l}4.445 \\
4.522\end{array}$ \\
\hline $\begin{array}{l}462 \mathrm{~A} \\
462 \mathrm{~A}\end{array}$ & $\begin{array}{l}43 \\
46\end{array}$ & $i$ & $115-125$ & $\begin{array}{l}3000 \\
1030\end{array}$ & $\begin{array}{l}\begin{array}{l}0430 \\
1130\end{array} \\
\text {. }\end{array}$ & $\begin{array}{l}23 \\
24\end{array}$ & $\begin{array}{l}19.2 \\
18\end{array}$ & $16^{-}$ & $19^{-}$ & $\begin{array}{l}19 \\
17.5\end{array}$ & $\bar{z}$ & $\begin{array}{l}38.2 \\
35.5\end{array}$ & $\begin{array}{l}20 \\
20\end{array}$ & $\begin{array}{l}8 \\
8\end{array}$ & $\begin{array}{l}\text { High } \\
\text { High }\end{array}$ & $\begin{array}{l}1.679 \\
1.369\end{array}$ & $\begin{array}{l}4.664 \\
3.803\end{array}$ & $\begin{array}{l}1.607 \\
1.319\end{array}$ & $\begin{array}{l}1.0124 \\
10124\end{array}$ & $\begin{array}{r}1.627 \\
.336\end{array}$ & $\begin{array}{l}4.522 \\
3.713\end{array}$ \\
\hline $462 \mathrm{~A}$ & 46 & 2 & $108-118$ & 1030 & 1150 & 24 & 18 & 17 & & 18 & $\bar{z}$ & 36 & 20 & 8 & $\begin{array}{l}\text { Mign } \\
\text { High }\end{array}$ & 1.788 & $\begin{array}{l}3.803 \\
4.967\end{array}$ & $\begin{array}{l}1.319 \\
1.721\end{array}$ & $\begin{array}{l}1.00124 \\
1.0124\end{array}$ & $\begin{array}{l}1.336 \\
1.742\end{array}$ & $\begin{array}{l}3.713 \\
4.843\end{array}$ \\
\hline $462 \mathrm{~A}$ & 46 & 4 & $9-20$ & 1030 & 1150 & 24 & 18 & 16 & 18 & 17 & - & 35 & 20 & 8 & High & 1.600 & 4.445 & 1.544 & 1.0124 & 1.563 & 4.346 \\
\hline $462 \mathrm{~A}$ & 46 & 5 & $76-86$ & 1030 & 1305 & 24 & 20 & 18 & 20 & 19 & - & 39 & 20 & 8 & High & 1.515 & 4.209 & 1.447 & 1.0124 & 1.465 & 4.072 \\
\hline $462 \mathrm{~A}$ & 48 & 1 & $105-115$ & 0130 & 0300 & 23 & 19.2 & - & - & 18.5 & - & 37.7 & 20 & 8 & High & 2.019 & 5.609 & 1.935 & 1.0124 & 1.958 & 5.445 \\
\hline $462 \mathrm{~A}$ & 48 & 4 & $60-70$ & 0130 & 0300 & 23 & 19.2 & - & - & 20.5 & - & 39.7 & 20 & 8 & High & 1.610 & 4.473 & 1.535 & 1.0124 & 1.554 & 4.319 \\
\hline $462 \mathrm{~A}$ & 49 & 1 & $74-84$ & 0550 & 0730 & 23 & 19.8 & - & - & 23.5 & - & 43.3 & 20 & 8 & High & 1.580 & 4.389 & 1.492 & 1.0124 & 1.511 & 4.199 \\
\hline $462 \mathrm{~A}$ & 49 & 3 & $0-10$ & 0550 & 0800 & 23 & 21 & 18 & 20 & 19 & - & 40 & 20 & 8 & High & 1.401 & 3.892 & 1.334 & 1.0124 & 1.350 & 3.755 \\
\hline $462 \mathrm{~A}$ & 50 & 1 & $15-24$ & 1150 & 1550 & 24 & 23.5 & 17 & 18.5 & 17.75 & - & 41.3 & 20 & 8 & High & 1.727 & 4.798 & 1.639 & 1.0124 & 1.659 & 4.613 \\
\hline $462 \mathrm{~A}$ & 50 & 6 & $2-12$ & 1150 & 1600 & 24 & 23.5 & 16 & 18 & 17 & - & 40.5 & 20 & 8 & High & 1.888 & 5.255 & $1.794 ?$ & 1.0124 & 1.817 & 5.049 \\
\hline $462 \mathrm{~A}$ & 50 & 6 & $120-130$ & 1150 & 1630 & 24 & 23.7 & 17 & 18 & 17.5 & - & 41.2 & 20 & 8 & High & 1.980 & 5.500 & 1.880 & 1.0124 & 1.904 & 5.292 \\
\hline $462 \mathrm{~A}$ & 51 & 3 & $\begin{array}{c}63-73 \\
0\end{array}$ & 1921 & 2115 & 24 & 20.4 & 19 & 21 & $\begin{array}{l}20 \\
17\end{array}$ & - & $\begin{array}{l}40.4 \\
35\end{array}$ & 20 & $\begin{array}{l}8 \\
8\end{array}$ & $\begin{array}{l}\text { High } \\
\text { High }\end{array}$ & $\begin{array}{l}1.695 \\
1609\end{array}$ & $\begin{array}{r}4.709 \\
\end{array}$ & 1.613 & 1.0124 & $\begin{array}{l}1.633 \\
570\end{array}$ & $\begin{array}{r}4.539 \\
\end{array}$ \\
\hline $462 \mathrm{~A}$ & 52 & $\frac{1}{2}$ & 126-136 & 0321 & 0431 & 24 & $\begin{array}{l}18 \\
18\end{array}$ & $\bar{z}$ & $=$ & $\begin{array}{l}17 \\
18\end{array}$ & $\overline{-}$ & 35 & 20 & $\begin{array}{l}8 \\
8\end{array}$ & $\begin{array}{l}\text { High } \\
\text { High }\end{array}$ & $\begin{array}{l}1.609 \\
1.638\end{array}$ & $\begin{array}{l}4.470 \\
4550\end{array}$ & 1.553 & 1.0124 & 1.572 & $\begin{array}{r}4.370 \\
\end{array}$ \\
\hline $\begin{array}{l}462 \mathrm{~A} \\
462 \mathrm{~A}\end{array}$ & $\begin{array}{l}52 \\
58\end{array}$ & 2 & $\begin{array}{c}113-123 \\
50-60\end{array}$ & $\begin{array}{l}0321 \\
1234\end{array}$ & 0431 & $\begin{array}{r}24 \\
24\end{array}$ & $\begin{array}{l}18 \\
19 \text { (est.) }\end{array}$ & $16^{-}$ & $17^{-}$ & $\begin{array}{l}18 \\
17.5\end{array}$ & $\overline{-}$ & $\begin{array}{l}36 \\
36.5\end{array}$ & 20 & $\begin{array}{l}8 \\
8\end{array}$ & $\begin{array}{l}\text { High } \\
\text { High }\end{array}$ & $\begin{array}{l}1.638 \\
2012\end{array}$ & $\begin{array}{l}4.550 \\
5.589\end{array}$ & $\begin{array}{l}1.577 \\
1.934\end{array}$ & $\begin{array}{l}1.0124 \\
10124\end{array}$ & $\begin{array}{l}1.596 \\
1.958\end{array}$ & $\begin{array}{l}4.436 \\
5.443\end{array}$ \\
\hline $\begin{array}{l}462 \mathrm{~A} \\
462 \mathrm{~A}\end{array}$ & $\begin{array}{l}58 \\
59\end{array}$ & 1 & $\begin{array}{l}50-60 \\
58-68\end{array}$ & $\begin{array}{l}1234 \\
2245\end{array}$ & 2400 & $\begin{array}{l}24 \\
23\end{array}$ & $\begin{array}{l}19 \text { (est.) } \\
19\end{array}$ & $\begin{array}{l}16 \\
15\end{array}$ & $\begin{array}{l}17 \\
17\end{array}$ & $\begin{array}{l}17.5 \\
16\end{array}$ & $=$ & $\begin{array}{l}36.5 \\
35\end{array}$ & $\begin{array}{l}20 \\
20\end{array}$ & $\begin{array}{l}8 \\
8\end{array}$ & $\begin{array}{l}\text { High } \\
\text { High }\end{array}$ & $\begin{array}{l}2.012 \\
1797\end{array}$ & $\begin{array}{l}5.589 \\
4.992\end{array}$ & $\begin{array}{l}1.9344 \\
1.734\end{array}$ & $\begin{array}{l}1.0124 \\
10124\end{array}$ & $\begin{array}{l}1.958 \\
1.756\end{array}$ & $\begin{array}{r}5.443 \\
4880\end{array}$ \\
\hline $\begin{array}{l}402 \mathrm{~A} \\
462 \mathrm{~A}\end{array}$ & 59 & 2 & $53-63$ & 2245 & 2400 & $\begin{array}{l}23 \\
23\end{array}$ & 19 & 16 & $\begin{array}{l}17 \\
19\end{array}$ & $\begin{array}{l}16 \\
18\end{array}$ & $\bar{z}$ & $\begin{array}{l}35 \\
37\end{array}$ & $\begin{array}{l}20 \\
20\end{array}$ & $\begin{array}{l}8 \\
8\end{array}$ & $\begin{array}{l}\text { Hilgh } \\
\text { High }\end{array}$ & $\begin{array}{l}1.797 \\
1.662\end{array}$ & $\begin{array}{l}4.992 \\
4.617\end{array}$ & $\begin{array}{l}1.774 \\
1.596\end{array}$ & $\begin{array}{l}1.0124 \\
10124\end{array}$ & $\begin{array}{l}1.756 \\
1.615\end{array}$ & $\begin{array}{l}4.880 \\
4.490\end{array}$ \\
\hline $462 \mathrm{~A}$ & 59 & 4 & $45-55$ & 2245 & 0300 & 23 & 20 & 17 & 19 & 18 & - & 38 & 20 & 8 & High & $\begin{array}{l}1.062 \\
1.687\end{array}$ & $\begin{array}{l}4.617 \\
4.686\end{array}$ & $\begin{array}{l}1.596 \\
1.615\end{array}$ & $\begin{array}{l}1.0124 \\
10124\end{array}$ & $\begin{array}{l}1.615 \\
1.635\end{array}$ & $\begin{array}{l}4.490 \\
4.545\end{array}$ \\
\hline $462 \mathrm{~A}$ & 61 & 1 & 28-38 & 0940 & 1135 & 24 & 20.4 & 17 & 18.5 & 17.75 & - & 38.2 & 20 & 8 & High & 1.591 & 4.420 & $\begin{array}{l}1.523 \\
1.523\end{array}$ & 1.0124 & $\begin{array}{l}1.630 \\
1.541\end{array}$ & $\begin{array}{l}4.545 \\
4.285\end{array}$ \\
\hline $462 \mathrm{~A}$ & 61 & 4 & $56-66$ & 0940 & 1200 & 24 & 21.4 & 23 & 25 & 24 & - & 45.4 & 20 & 8 & High & 1.519 & 4.214 & 1.426 & 1.0124 & 1.444 & 4.014 \\
\hline $462 \mathrm{~A}$ & 62 & 2 & $50-60$ & 1525 & 1620 & $24 ?$ & 18 & 17 & 19 & 18 & - & 36 & 20 & 8 & High & 1.615 & 4.486 & 1.554 & 1.0124 & 1.573 & 4.378 \\
\hline $462 \mathrm{~A}$ & 64 & 2 & $3-13$ & 1145 & 1300 & $24 ?$ & 18.8 & - & - & 17.5 & - & 36.3 & 20 & 8 & High & 2.106 & 5.85 & 2.025 & 1.0124 & 2.050 & 5.700 \\
\hline $462 \mathrm{~A}$ & 65 & 1 & $6-16$ & 1710 & 1100 & 24 & 23.8 & - & - & - & $20 ?$ & 43.8 & 20 & 8 & High & 1.777 & 4.937 & 1.676 & 1.0124 & 1.696 & 4.716 \\
\hline $462 \mathrm{~A}$ & 65 & 2 & $89-99$ & 1710 & 1100 & 24 & 23.8 & - & - & - & $20 ?$ & 43.8 & 20 & 8 & High & 1.728 & 4.800 & 1.630 & 1.0124 & 1.650 & 4.586 \\
\hline $462 \mathrm{~A}$ & 66 & 1 & $138-148$ & 2340 & 0045 & 24 & 18.3 & 19.5 & 21 & 20 & - & 38.3 & 20 & 8 & High & 1.839 & 5.109 & 1.759 & 1.0124 & 1.780 & 4.951 \\
\hline $462 \mathrm{~A}$ & 66 & 2 & $123-133$ & 2340 & 0045 & 24 & 18.3 & 17.5 & 19.5 & 18.5 & - & 36.8 & 20 & 8 & High & 1.627 & 4.520 & 1.563 & 1.0124 & 1.582 & $\begin{array}{l}4.398 \\
.308\end{array}$ \\
\hline $462 \mathrm{~A}$ & 66 & 4 & $90-100$ & 2340 & 0045 & 24 & 18.3 & 16 & 17.5 & 16.8 & - & 35.1 & 20 & 8 & High & 1.910 & 5.306 & 1.843 & 1.0124 & 1.865 & 5.186 \\
\hline $462 \mathrm{~A}$ & 66 & 5 & 53-63 & 2340 & 0045 & 24 & 18.3 & $\begin{array}{l}16.5 \\
165\end{array}$ & $\begin{array}{l}18 \\
18\end{array}$ & $\begin{array}{l}17.3 \\
17.3\end{array}$ & $\overline{-}$ & $\begin{array}{l}35.6 \\
356\end{array}$ & 20 & 8 & High & 1.886 & 5.239 & 1.817 & 1.0124 & $\begin{array}{l}1.840 \\
807\end{array}$ & 5.115 \\
\hline $462 \mathrm{~A}$ & 66 & 6 & 63-73 & $\begin{array}{l}2340 \\
2340\end{array}$ & 0045 & 24 & 18.3 & 16.5 & 18 & 17.3 & - & 35.6 & 20 & $\begin{array}{l}8 \\
8\end{array}$ & $\begin{array}{l}\text { High } \\
\text { High }\end{array}$ & 1.873 & $\begin{array}{r}5.203 \\
\end{array}$ & 1.805 & 1.0124 & 1.827 & $\begin{array}{l}5.079 \\
4.808\end{array}$ \\
\hline $462 \mathrm{~A}$ & $\begin{array}{l}66 \\
67\end{array}$ & $?$ & $\begin{array}{r}1-10 \\
12-10\end{array}$ & $\begin{array}{l}2340 \\
05515\end{array}$ & $\begin{array}{l}0045 \\
0630\end{array}$ & 24 & $\begin{array}{l}18.3 \\
18.8\end{array}$ & $\begin{array}{l}15 \\
165\end{array}$ & $\begin{array}{l}17 \\
18\end{array}$ & $\begin{array}{l}16 \\
17\end{array}$ & $\bar{z}$ & $\begin{array}{l}34.3 \\
35.8\end{array}$ & 20 & $\begin{array}{l}8 \\
8\end{array}$ & $\begin{array}{l}\text { High } \\
\text { High }\end{array}$ & $\begin{array}{l}1.767 \\
1.745\end{array}$ & $\begin{array}{r}4.909 \\
\end{array}$ & 1.708 & 1.0124 & $\begin{array}{l}1.730 \\
\end{array}$ & $\begin{array}{l}4.808 \\
4.730\end{array}$ \\
\hline $\begin{array}{l}462 \mathrm{~A} \\
462 \mathrm{~A}\end{array}$ & $\begin{array}{l}67 \\
67\end{array}$ & $\begin{array}{l}1 \\
3\end{array}$ & & $\begin{array}{l}05515 \\
0515\end{array}$ & $\begin{array}{l}0630 \\
0630\end{array}$ & ${ }_{24}^{24}$ & $\begin{array}{l}18.8 \\
18.8\end{array}$ & $\begin{array}{l}16.5 \\
14.5\end{array}$ & $\begin{array}{l}18 \\
16.5\end{array}$ & $\begin{array}{l}17 \\
15\end{array}$ & $\overline{-}$ & $\begin{array}{l}33.8 \\
33.8\end{array}$ & 20 & $\begin{array}{l}8 \\
8\end{array}$ & $\begin{array}{l}\text { High } \\
\text { High }\end{array}$ & $\begin{array}{l}1.745 \\
1.904\end{array}$ & $\begin{array}{l}4.848 \\
5.289\end{array}$ & 1.680 & $\begin{array}{l}1.0124 \\
10124\end{array}$ & $\begin{array}{l}1.701 \\
1.865\end{array}$ & $\begin{array}{l}4.730 \\
5.187\end{array}$ \\
\hline $\begin{array}{l}462 \mathrm{~A} \\
462 \mathrm{~A}\end{array}$ & $\begin{array}{l}67 \\
67\end{array}$ & 7 & $\begin{array}{l}0-10 \\
10-20\end{array}$ & $\begin{array}{l}0515 \\
0515\end{array}$ & $\begin{array}{l}00630 \\
0630\end{array}$ & $\begin{array}{l}24 \\
24\end{array}$ & $\begin{array}{l}18.8 \\
18.8\end{array}$ & $\begin{array}{l}14.5 \\
16\end{array}$ & $\begin{array}{l}16.5 \\
18\end{array}$ & $\begin{array}{l}15 \\
17\end{array}$ & $\overline{-}$ & $\begin{array}{l}33.8 \\
35.8\end{array}$ & $\begin{array}{l}20 \\
20\end{array}$ & $\begin{array}{l}8 \\
8\end{array}$ & $\begin{array}{l}\text { High } \\
\text { High }\end{array}$ & $\begin{array}{l}1.904 \\
1.727\end{array}$ & $\begin{array}{r}5.289 \\
4.798\end{array}$ & $\begin{array}{l}1.843 \\
1.663\end{array}$ & $\begin{array}{l}1.00124 \\
10124\end{array}$ & $\begin{array}{l}1.865 \\
1.684\end{array}$ & $\begin{array}{l}5.187 \\
4.681\end{array}$ \\
\hline $462 \mathrm{~A}$ & 68 & 1 & $44-54$ & 1256 & 1350 & 24 & 17.8 & - & ${ }^{18}-$ & 17 & $\overline{-}$ & 34.8 & 20 & 8 & $\begin{array}{l}\text { High } \\
\text { High }\end{array}$ & $\begin{array}{l}1.827 \\
1.892\end{array}$ & $\begin{array}{l}4.798 \\
5.256\end{array}$ & $\begin{array}{l}1.663 \\
1.827\end{array}$ & $\begin{array}{l}1.00124 \\
1.0124\end{array}$ & $\begin{array}{l}1.684 \\
1.849\end{array}$ & $\begin{array}{l}4.681 \\
5.141\end{array}$ \\
\hline $462 \mathrm{~A}$ & 68 & 2 & $70-80$ & 1256 & 1400 & 24 & 18.2 & - & - & 18 & - & 36.2 & 20 & 8 & High & 1.781 & 4.948 & 1.713 & 1.0124 & 1.735 & 4.822 \\
\hline $462 \mathrm{~A}$ & 68 & 4 & $103-113$ & 1256 & 1515 & 24 & 21.3 & - & - & 18 & - & 39.3 & 20 & 8 & High & 1.855 & 5.236 & 1.799 & 1.0124 & 1.822 & 5.063 \\
\hline $462 \mathrm{~A}$ & 70 & 1 & $115-125$ & 0227 & 0400 & 24 & 19.3 & 18 & 20 & 19 & - & 38.3 & 20 & 8 & High & 1.649 & 4.581 & 1.578 & 1.0124 & 1.597 & 4.438 \\
\hline $462 \mathrm{~A}$ & 71 & 1 & $44-54$ & 0550 & 0700 & 24 & 18.6 & 16 & 18 & 17 & - & 35.6 & 20 & 8 & High & 1.553 & 4.314 & 1.496 & 1.0124 & 1.515 & 4.212 \\
\hline $462 \mathrm{~A}$ & 74 & 1 & $1-11$ & 0845 & $?$ & 24 & 18.6 & - & - & 18 & - & 36.6 & 20 & 8 & High & 1.591 & 4.420 & 1.529 & 1.0124 & 1.548 & 4.304 \\
\hline $462 \mathrm{~A}$ & 74 & 2 & $75-85$ & 0845 & $?$ & 24 & 18.6 & - & - & 16 & - & 34.6 & 20 & 8 & High & 1.780 & 4.945 & 1.719 & 1.0124 & 1.740 & 4.839 \\
\hline $462 \mathrm{~A}$ & 74 & 3 & $119-130$ & 0845 & $?$ & 24 & 18.6 & - & - & 18 & - & 36.6 & 20 & 8 & High & 1.895 & 5.265 & 1.821 & 1.0124 & 1.844 & 5.125 \\
\hline $462 \mathrm{~A}$ & 74 & 5 & $90-100$ & 0845 & $?$ & 24 & 18.6 & - & - & 17 & - & 35.6 & 20 & 8 & High & 1.534 & 4.261 & 1.478 & 1.0124 & 1.496 & 4.160 \\
\hline $462 \mathrm{~A}$ & 75 & 2 & 28-38 & 2200 & 0300 & 21.5 & 21.5 & - & - & - & 8.5 & 30 & 40 & 4 & High & 1.737 & 3.088 & 1.698 & 1.0325 & 1.753 & 4.873 \\
\hline $462 \mathrm{~A}$ & 75 & 2 & $7-17$ & 2200 & 0300 & 21.5 & 21.5 & - & $0^{-}$ & $\overline{85}$ & 8.5 & 30 & 40 & 4 & High & 1.907 & $\begin{array}{l}3.391 \\
.775\end{array}$ & 1.864 & 1.0325 & 1.924 & $\begin{array}{r}5.350 \\
\end{array}$ \\
\hline $462 \mathrm{~A}$ & 76 & 1 & $40-50$ & 0900 & 1200 & 21.5 & 20 & 8 & 9 & 8.5 & $\overline{0}$ & 28.5 & 20 & 4 & High & 1.719 & 4.775 & 1.687 & 1.0325 & 1.741 & $\begin{array}{l}4.842 \\
4578\end{array}$ \\
\hline $462 \mathrm{~A}$ & 77 & 1 & $\begin{array}{l}18-28 \\
68-78\end{array}$ & 1612 & $\begin{array}{l}1730 \\
0770\end{array}$ & 21.5 & 16.5 & $=$ & - & $\bar{z}$ & 9 & $\begin{array}{l}25.5 \\
25.5\end{array}$ & 20 & 4 & $\begin{array}{l}\text { High } \\
\text { High }\end{array}$ & 1.613 & $\begin{array}{r}4.481 \\
4.48\end{array}$ & 1.595 & $\begin{array}{l}1.0325 \\
1.0325\end{array}$ & $\begin{array}{l}1.646 \\
\end{array}$ & $\begin{array}{l}4.578 \\
4.748\end{array}$ \\
\hline $\begin{array}{l}462 \mathrm{~A} \\
462 \mathrm{~A}\end{array}$ & $\begin{array}{l}77 \\
78\end{array}$ & 2 & $\begin{array}{l}68-78 \\
88-98\end{array}$ & 1612 & $\begin{array}{l}1730 \\
0530\end{array}$ & $\begin{array}{l}21.5 \\
21.5\end{array}$ & $\begin{array}{l}16.5 \\
20.5\end{array}$ & $8^{-}$ & $9 \overline{5}$ & $\overline{8.8}$ & 9 & $\begin{array}{l}25.5 \\
29.5\end{array}$ & ${ }_{20}^{20}$ & $\begin{array}{l}4 \\
4\end{array}$ & $\begin{array}{l}\text { High } \\
\text { High }\end{array}$ & $\begin{array}{l}1.673 \\
1.650\end{array}$ & $\begin{array}{l}4.148 \\
4.84\end{array}$ & $\begin{array}{l}1.654 \\
1.615\end{array}$ & $\begin{array}{l}1.0325 \\
1.0325\end{array}$ & $\begin{array}{l}1.708 \\
1.67\end{array}$ & $\begin{array}{l}4.748 \\
4.635\end{array}$ \\
\hline $\begin{array}{l}462 \mathrm{~A} \\
462 \mathrm{~A}\end{array}$ & $\begin{array}{l}78 \\
78\end{array}$ & $\begin{array}{l}1 \\
2\end{array}$ & $\begin{array}{c}88-98 \\
79-89\end{array}$ & $\begin{array}{l}0017 \\
0017\end{array}$ & $\begin{array}{l}0530 \\
0530\end{array}$ & $\begin{array}{l}21.5 \\
21.5\end{array}$ & $\begin{array}{l}20.5 \\
20.5\end{array}$ & $\begin{array}{l}8 \\
8.5\end{array}$ & $\begin{array}{l}9.5 \\
9.5\end{array}$ & $\begin{array}{l}8.8 \\
9\end{array}$ & $\overline{-}$ & $\begin{array}{l}29.5 \\
29.5\end{array}$ & $\begin{array}{l}20 \\
20\end{array}$ & $\begin{array}{l}4 \\
4\end{array}$ & $\begin{array}{l}\text { High } \\
\text { High }\end{array}$ & $\begin{array}{l}1.650 \\
1.614\end{array}$ & $\begin{array}{l}4.584 \\
4.484\end{array}$ & $\begin{array}{l}1.615 \\
1580\end{array}$ & $\begin{array}{l}1.0325 \\
10325\end{array}$ & $\begin{array}{r}1.667 \\
1.631\end{array}$ & $\begin{array}{l}4.635 \\
4.535\end{array}$ \\
\hline $\begin{array}{l}462 \mathrm{~A} \\
462 \mathrm{~A}\end{array}$ & $\begin{array}{l}18 \\
79\end{array}$ & $\begin{array}{l}2 \\
1\end{array}$ & $\begin{array}{c}73-89 \\
134-144 ?\end{array}$ & $\begin{array}{l}0017 \\
0615\end{array}$ & $\begin{array}{l}0.030 \\
1015\end{array}$ & $\begin{array}{l}21.5 \\
21.5\end{array}$ & $\begin{array}{l}20.5 \\
20.5\end{array}$ & $\begin{array}{l}8.5 \\
8\end{array}$ & 9 & 8.5 & $\overline{-}$ & 29 & $\begin{array}{l}20 \\
20\end{array}$ & $\begin{array}{l}4 \\
4\end{array}$ & $\begin{array}{l}\text { High } \\
\text { High }\end{array}$ & $\begin{array}{l}1.614 \\
1.579\end{array}$ & $\begin{array}{l}4.484 \\
4.386\end{array}$ & $\begin{array}{l}1.580 \\
1.547\end{array}$ & $\begin{array}{l}1.0325 \\
1.0325\end{array}$ & $\begin{array}{l}1.631 \\
1.598\end{array}$ & $\begin{array}{l}4.535 \\
4.442\end{array}$ \\
\hline $462 \mathrm{~A}$ & 79 & 2 & $131-141$ ? & 0615 & 1015 & 21.5 & 20.5 & - & . & 8.5 & - & 29 & 20 & 4 & High & 1.590 & 4.417 & 1.558 & 1.0325 & 1.608 & 4.471 \\
\hline $462 \mathrm{~A}$ & 79 & 5 & 38-48? & 0615 & 1015 & 21.5 & 20.5 & 8.5 & 9.5 & 9 & - & 30 & 20 & 4 & High & 1.740 & 4.834 & 1.701 & 1.0325 & 1.756 & 4.882 \\
\hline $462 \mathrm{~A}$ & 80 & 1 & $26-36 ?$ & 1112 & 1630 & 21.5 & 21 & 8 & 10 & 9 & - & 30 & 20 & 4 & High & 1.221 & 3.392 & 1.194 & 1.0325 & 1.232 & 3.426 \\
\hline
\end{tabular}




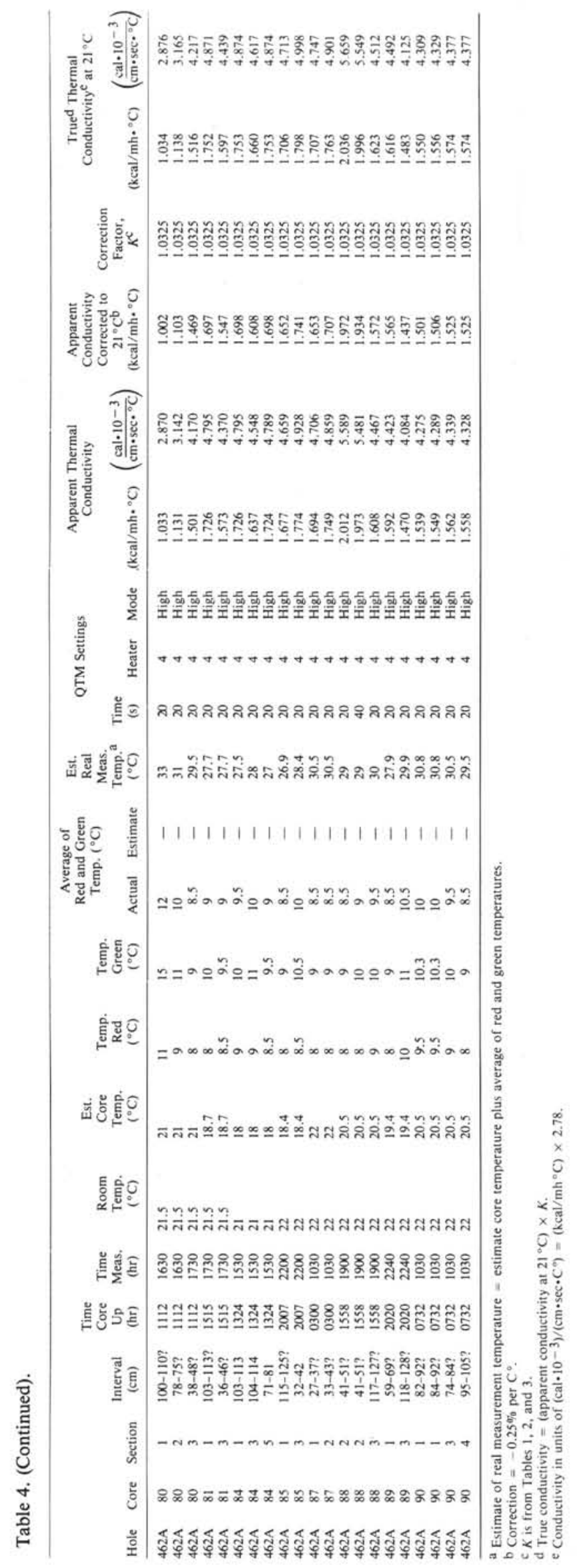

late true density we have to determine $\mu_{\mathrm{a}}$ by linear interpolation between the $6.61-\mathrm{cm}$ aluminum standard and $2.54-\mathrm{cm}$ aluminum standard, based on the thickness of the sample. We will assign a value of $0.1080 \mathrm{~cm}^{2} / \mathrm{g}$ to the $6.61-\mathrm{cm}$ aluminum standard and $0.1104 \mathrm{~cm}^{2} / \mathrm{g}$ to the $2.54-\mathrm{cm}$ aluminum standard (averages are in Table 13) for Site 462

The 2-minute GRAPE technique and calculations used on Leg 61 are summarized as follows: for each GRAPE 2-minute count determination we measured the gamma count $(2 \mathrm{~min})$ through the sample, with a new gamma count $(2 \mathrm{~min})$ in air for each sample, and measured the sample's thickness. We then used these values in Equation 24 (Boyce, 1976a) to calculate the Evans (1965) corrected wet-bulk density ( $\left.\varrho_{\mathrm{bc}}\right)$, using a $\mu_{\mathrm{a}}$ derived from linear interpolation (as discussed in Boyce, 1976a) between (1) $0.1080 \mathrm{~cm}^{2} / \mathrm{g}$ assigned to the $6.61-\mathrm{cm}$ aluminum standard and (2) $0.1104 \mathrm{~cm}^{2} / \mathrm{g}$ assigned to the $2.54-\mathrm{cm}$ aluminum standard. We then took this $\varrho_{b c}$, derived from Equation 24, and placed it into Equation 21 (Boyce, 1976a) to calculate "true" wet-bulk density. The following numerical data were used in Equation 21: (1) grain density $\left(\varrho_{\mathrm{g}}\right)=2.7 \mathrm{~g} / \mathrm{cm}^{3}$ for sedimentary rock and $3.0 \mathrm{~g} / \mathrm{cm}^{3}$ for basalt; (2) corrected grain density $\left(\varrho_{\mathrm{gc}}\right)=2.7 \mathrm{~g} / \mathrm{cm}^{3}$ for sedimentary rock and $3.0 \mathrm{~g} / \mathrm{cm}^{3}$ for basalt; (3) fluid density $\left(\varrho_{\mathrm{f}}\right)=$ $1.025 \mathrm{~g} / \mathrm{cm}^{3}$; and $(4)$ corrected fluid density $\left(\varrho_{\mathrm{fc}}\right)=$ $1.128 \mathrm{~g} / \mathrm{cm}^{3}$. This final "true"' wet-bulk density derived from Equation 21 is the value published in the Leg 50 volume. See Boyce (1976a) for discussions of errors and assumptions of these formulas.

The GRAPE data assume that the sediments and rocks have a $35 \%$ interstitial-water salinity.

\section{Gravimetric Wet-Bulk Density, Wet-Water Content, and Porosity}

On the Glomar Challenger 20- to 30-gram samples were processed by the shipboard technicians to determine gravimetrically the wet-bulk density, wet-water content, and porosity.

Wet-bulk density is the ratio of the "mass of wetsaturated geologic sample" to "its volume," in units of grams per cubic $\mathrm{cm}\left(\mathrm{g} / \mathrm{cm}^{3}\right)$. Wet-water content is the ratio of the "weight of sea water in a geologic sample" to the "weight of the wet-saturated geologic sample," expressed as a percentage. Porosity is the ratio of "the volume of pore space in a geologic sample" to the "volume of the wet-saturated sample," reported as a percentage.

On board the ship, the 20- to 30 -gram samples were weighed in air and weighed after submersion in water, using an OHAUS Centrogram Triple Beam (311 gram) Balance, then dried in the oven at $110^{\circ} \mathrm{C}$ for 24 hours, cooled in a desiccator for at least 2 hours, and then reweighed. Salt corrections for $35 \%$ salinities (as described in Boyce, 1976a) are applied to the valued wetwater content, and porosity. See Rocker (1974) for discussion of this technique.

Tables 14 and 15 show the reproducibility of method in terms of wet-bulk density $\left( \pm 0.005 \mathrm{~g} / \mathrm{cm}^{3}\right)$, which is about $\pm 0.2 \%$ (absolute) precision for the porosity and water-content values: 


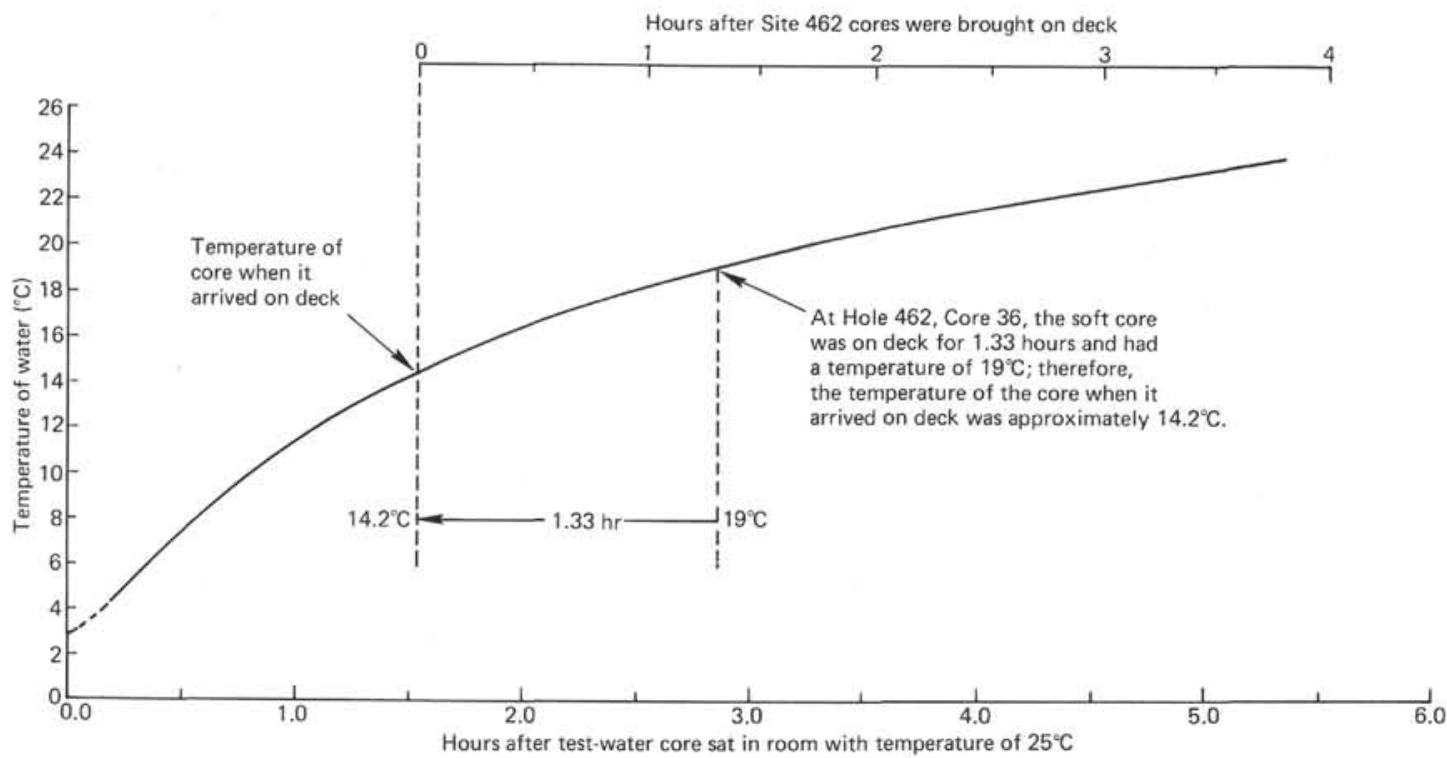

Figure 1. The upper time-versus-temperature curve used to determine minimum core temperatures of the thermal conductivity (Table 4 ) and sonic-velocity time-temperature measurements.

Table 5. Correction factors for the Tektronics 835 oscilloscope, DSDP Leg 61.

\begin{tabular}{cc}
\hline $\begin{array}{c}\text { Scope Setting } \\
\mu \mathrm{S} / \mathrm{cm}\end{array}$ & $K$ \\
\hline 1.0 & Apparent velocity $\times 1.0108=$ true velocity \\
2.0 & Apparent velocity $\times 1.0095=$ true velocity \\
5.0 & Apparent velocity $\times 1.0077=$ true velocity \\
\hline
\end{tabular}

Table 6. Predetermined sound velocities of lucite, brass, and aluminum semi-standards, as listed in Boyce (1973).

\begin{tabular}{clll}
\hline \multicolumn{1}{c}{ Author } & \multicolumn{1}{c}{ Lucite } & \multicolumn{1}{c}{ Brass } & \multicolumn{1}{c}{ Aluminum } \\
\hline Boyce (1973) & $2.741 \mathrm{~km} / \mathrm{s}$ & $4.506 \mathrm{~km} / \mathrm{s}$ & $6.293 \mathrm{~km} / \mathrm{s}$ \\
& $( \pm 0.84 \%)$ & $( \pm 0.45 \%)$ & $( \pm 1.29 \%)$ \\
Schreiber $^{\mathrm{a}}$ & $2.745 \mathrm{~km} / \mathrm{s}$ & $4.529 \mathrm{~km} / \mathrm{s}$ & $6.295 \mathrm{~km} / \mathrm{s}$ \\
& $( \pm 0.006 \mathrm{~km} / \mathrm{s})$ & $( \pm 0.004 \mathrm{~km} / \mathrm{s})$ & $( \pm 0.008 \mathrm{~km} / \mathrm{s})$ \\
\hline
\end{tabular}

${ }^{a}$ Lamont-Doherty Geological Observatory (pers. comm., 1971). Schreiber used the modified pulse-transmission method (Mottaboni and Schreiber, 1967).

1) Wet-bulk density (with pan weights removed) is equal to the weight of saturated sample in air, divided by its volume. Volume is equal to weight in air minus weight in water, divided by $1.00 \mathrm{~g} / \mathrm{cm}^{3}$.

2) Wet-water content (with pan weights removed), without salt correction is equal to the weight of evaporated water divided by the weight of the saturated sample. The weight of evaporated water is equal to the weight of the saturated sample minus the weight of the dried sample.

Salt corrected wet-water content $(\%)$ is:

$$
\left[\frac{\frac{\text { (weight evaporated water) }}{(0.965)}}{\overline{\text { weight saturated sample }}}\right] \times 100
$$

Table 7. Uncorrected velocities $(\mathrm{km} / \mathrm{s})$ through distilled water at $23^{\circ} \mathrm{C}$.

\begin{tabular}{lcc}
\hline Scope Setting ${ }^{\mathrm{a}, \mathrm{b}, \mathrm{c}}$ & $2.0 \mu \mathrm{s} / \mathrm{cm}$ & $5.0 \mu \mathrm{s} / \mathrm{cm}$ \\
Thickness of Water & $0.957 \mathrm{~cm}$ & $2.50 \mathrm{~cm}$ \\
\hline & $1.480^{\mathrm{b}}$ & $1.486^{\mathrm{b}}$ \\
& 1.495 & 1.492 \\
& 1.491 & 1.476 \\
& 1.486 & 1.493 \\
& 1.497 & 1.495 \\
& 1.498 & 1.488 \\
& 1.498 & 1.489 \\
& 1.500 & 1.494 \\
Average & 1.478 & 1.489 \\
Range & 1.480 & 1.486 \\
$K^{\mathrm{d}}$ & 1.490 & 1.488 \\
& $+0.7 \%$ & $+0.4 \%$ \\
\hline
\end{tabular}

The best precision is obtained when the entire range on the $\mu \mathrm{s} / \mathrm{cm}$ delay dial is used:

a Good use: 6-10 range.

b Fair use: $3-6$ range.

c Poor use: $0-3$ range.

d $K=$ true velocity/average apparent velocity. True velocity $=K \times$ average apparent velocity $=1.502 \mathrm{~km} / \mathrm{s}$.

where the weight of sea water is equal to the weight of the evaporated water divided by 0.965 .

3) Porosity $(\%)$ without salt corrections (with pan weights removed) is:

$$
\left[\frac{\frac{\text { (weight evaporated water) }}{1.00 \mathrm{~g} / \mathrm{cm}^{3}}}{\overline{\text { (volume of saturated sample) }}}\right] \times 100
$$

where sample volume is equal to the weight of the 
Table 8 . Uncorrected velocity $(\mathrm{km} / \mathrm{s})$ through lucite sonic semi-standards.

\begin{tabular}{|c|c|c|c|c|}
\hline \multirow{2}{*}{$\begin{array}{l}\text { Scope Setting }{ }^{a, b}, c \\
\text { Thickness of semi-standards }\end{array}$} & \multicolumn{2}{|c|}{$2.0 \mu \mathrm{s} / \mathrm{cm}$} & \multicolumn{2}{|c|}{$5.0 \mu \mathrm{s} / \mathrm{cm}$} \\
\hline & $2.54 \mathrm{~cm}$ & $5.00 \mathrm{~cm}$ & $2.54 \mathrm{~cm}$ & $5.00 \mathrm{~cm}$ \\
\hline & $2.740^{b}$ & - & $2.720^{\mathrm{c}}$ & $2.720^{b}$ \\
\hline & 2.737 & - & 2.748 & 2.733 \\
\hline & 2.737 & - & 2.748 & 2.735 \\
\hline & 2.728 & - & 2.755 & 2.726 \\
\hline & 2.745 & - & 2.769 & 2.723 \\
\hline & 2.727 & - & 2.739 & 2.737 \\
\hline & 2.717 & - & 2.747 & 2.729 \\
\hline & 2.725 & - & 2.711 & 2.728 \\
\hline & 2.725 & - & 2.711 & 2.723 \\
\hline & 2.728 & - & 2.748 & 2.730 \\
\hline Average & 2.731 & - & 2.740 & 2.728 \\
\hline Range $\{+\%$ & $+0.5 \%$ & - & $+0.5 \%$ & $+0.3 \%$ \\
\hline Kange $\left\{-\%_{0}\right.$ & $-0.5 \%$ & - & $-1.0 \%$ & $-0.3 \%$ \\
\hline$K^{\mathrm{d}}=$ & 1.0048 & - & 1.0015 & 1.0059 \\
\hline
\end{tabular}

saturated sample in air minus the weight of the saturated sample in water, and $1.00 \mathrm{~g} / \mathrm{cm}^{3}$ is the density of water.

Salt corrected porosity $(\%)$ is:

$$
\left[\frac{\frac{\text { (weight evaporated water } \div 0.965)}{1.0245 \mathrm{~g} / \mathrm{cm}^{3}}}{\overline{\text { (volume of saturated sample) }}}\right] \times 100
$$

where $1.0245 \mathrm{~g} / \mathrm{cm}^{3}$ is the fluid density of the sea-water salt, and the weight of sea water is equal to the weight of the evaporated water divided by 0.965 .

Four of the samples were processed by the cylinder technique. This technique uses a cylinder $(\sim 3-\mathrm{cm}$ radius, 2 -cm height, and $\sim 1$-mm thickness, with beveled edges), which is inserted into the soft sediment; it is then cut from the core, and the excess sediment is carefully cut off the cylinder. Then plastic plates are on the top and bottom of the cylinder. With rubber bands placed around it, the plastic plate and the sample is placed under sea water and shipped to the DSDP shore laboratory. There, the pre-weighed cylinders and plates plus the saturated sample are weighed; then the cylinder and plate are placed in an oven at $110^{\circ} \mathrm{C}$ for 24 hours, then placed in a desiccator to cool for at least 2 hours, then reweighed. Wet-water content, wet-bulk density, and porosity are calculated. The volume used in wetbulk density and porosity measurement is that of the cylinders.

Salt corrections are applied as discussed above. Precision is about $\pm 0.5 \%$.

One advantage of the cylinder technique is that the cylinder-technique sample also can be processed by the GRAPE 2-minute count method.

This is done as discussed for the GRAPE 2-minute wet-bulk density, but substituting the gamma-ray count through the two plastic plates for the air count. Then the cylinder with sample and the two plastic plates is counted for 2 minutes through the cylinder axis and through the two plastic plates. A 2-minute GRAPE wetbulk density can be calculated.

\section{GEARHART-OWEN WELL-LOG EQUIPMENT USED DURING LEG 61}

\section{Introduction}

During Leg 61, we used Gearhart-Owen Industries wire-line well-logging tools. Gearhart-Owen logs were grouped in each single lowering in Hole 462 as follows:

1) Absolute ${ }^{3}$ and differential temperature log, ${ }^{4} 3.65$ $\mathrm{cm}$ in diameter (successful).

\footnotetext{
${ }^{3}$ This is not "absolute temperature," i.e., Kelvin, in the scientific sense, but the formal name of the tool.

${ }_{4}^{4}$ Measured temperature on the way down and GR on the way up through the pipe to the sea floor.
}

Table 9. Uncorrected velocities $(\mathrm{km} / \mathrm{s})$ through brass sonic semi-standards.

\begin{tabular}{|c|c|c|c|c|c|c|}
\hline \multirow{2}{*}{$\begin{array}{l}\text { Scope Setting }{ }^{a, b}, c \\
\text { Thickness of Semi-standards }\end{array}$} & \multicolumn{2}{|c|}{$1.0 \mu \mathrm{s} / \mathrm{cm}$} & \multicolumn{2}{|c|}{$2.0 \mu \mathrm{s} / \mathrm{cm}$} & \multicolumn{2}{|c|}{$5.0 \mu \mathrm{s} / \mathrm{cm}$} \\
\hline & $2.54 \mathrm{~cm}$ & $5.00 \mathrm{~cm}$ & $2.54 \mathrm{~cm}$ & $5.00 \mathrm{~cm}$ & $2.54 \mathrm{~cm}$ & $5.00 \mathrm{~cm}$ \\
\hline & $4.549^{\mathrm{b}}$ & - & $4.474^{c}$ & $4.468^{b}$ & $4.622^{c}$ & $4.496^{c}$ \\
\hline & 4.497 & - & 4.435 & 4.471 & 4.472 & 4.461 \\
\hline & 4.492 & - & 4.460 & 4.465 & 4.627 & 4.482 \\
\hline & 4.483 & - & 4.489 & 4.444 & 4.601 & 4.530 \\
\hline & 4.464 & - & 4.447 & 4.463 & 4.552 & 4.458 \\
\hline & 4.407 & - & 4.458 & 4.456 & 4.472 & 4.483 \\
\hline & 4.439 & - & 4.500 & 4.487 & 4.563 & 4.448 \\
\hline & 4.444 & - & 4.515 & 4.471 & 4.492 & 4.486 \\
\hline & 4.485 & - & 4.461 & 4.475 & 4.584 & 4.595 \\
\hline & 4.493 & - & 4.447 & 4.476 & 4.581 & 4.450 \\
\hline Average & 4.475 & - & 4.469 & 4.468 & 4.557 & 4.489 \\
\hline Range $\left\{\begin{array}{l}+\% \\
-\%\end{array}\right.$ & $\begin{array}{l}+1.6 \% \\
-1.5 \%\end{array}$ & - & $\begin{array}{l}+1.0 \% \\
-0.8 \%\end{array}$ & $\begin{array}{l}+0.4 \% \\
-0.5 \%\end{array}$ & $\begin{array}{l}+1.5 \% \\
-1.9 \%\end{array}$ & $\begin{array}{l}+2.4 \% \\
-0.9 \%\end{array}$ \\
\hline$K^{\mathrm{d}}=$ & 1.0121 & - & 1.0134 & 1.0137 & 0.9939 & $1.008 \mathrm{~s}$ \\
\hline
\end{tabular}

The best precision occurs when entire range on the $\mu \mathrm{s} / \mathrm{cm}$ delay dial is used:

a Good use: $6-10$ range.

bair use: 3-6 range.

c Poor use: $0-3$ range.

$\mathrm{d}_{K=\text { true velocity/average apparent velocity. True velocity }=K \times \text { average apparent velocity }=4.529}$

$\mathrm{km} / \mathrm{s}$. 
Table 10. Uncorrected velocities $(\mathrm{km} / \mathrm{s})$ through aluminum sonic semi-standards.

\begin{tabular}{lcccccc}
\hline \multirow{2}{*}{$\begin{array}{l}\text { Scope Setting } \mathrm{a}, \mathrm{b}, \mathrm{c} \\
\text { Thickness of Semi-standards }\end{array}$} & \multicolumn{2}{c}{$1.0 \mu \mathrm{s} / \mathrm{cm}$} & \multicolumn{2}{c}{$2.0 \mu \mathrm{s} / \mathrm{cm}$} & \multicolumn{2}{c}{$5.0 \mu \mathrm{s} / \mathrm{cm}$} \\
& $2.54 \mathrm{~cm}$ & $5.00 \mathrm{~cm}$ & $2.54 \mathrm{~cm}$ & $5.00 \mathrm{~cm}$ & $2.54 \mathrm{~cm}$ & $5.00 \mathrm{~cm}$ \\
& $6.215^{\mathrm{b}}$ & - & $6.212^{\mathrm{c}}$ & $6.246^{\mathrm{b}}$ & $6.271^{\mathrm{c}}$ & $6.186^{\mathrm{c}}$ \\
& 6.261 & - & 6.169 & 6.269 & 6.310 & 6.158 \\
& 6.227 & - & 6.064 & 6.233 & 6.076 & 6.345 \\
& 6.253 & - & 6.190 & 6.266 & 6.308 & 6.316 \\
& 6.207 & - & 6.293 & 6.206 & 6.193 & 6.256 \\
& 6.265 & - & 6.183 & 6.152 & 6.300 & 6.350 \\
& 6.180 & - & 6.178 & 6.215 & 6.294 & 6.291 \\
& 6.197 & - & 6.215 & 6.210 & 6.195 & 6.249 \\
Average & 6.253 & - & 6.192 & 6.249 & 6.358 & 6.291 \\
Range $\left\{\begin{array}{l}+\% \\
-\%\end{array}\right.$ & 6.303 & - & 6.163 & 6.208 & 6.276 & 6.213 \\
$K^{\mathrm{d}}=$ & 6.236 & - & 6.186 & 6.225 & 6.258 & 6.266 \\
\hline
\end{tabular}

The best precision occurs when entire range on the $\mu \mathrm{s} / \mathrm{cm}$ delay dial is used:

a Good use: 6-10 range.

b Fair use: 3-6 range.

c Poor use: $0-3$ range.

d $K=$ true velocity/average apparent velocity. True velocity $=K \times$ average apparent velocity $=6.295$ $\mathrm{km} / \mathrm{s}$.

Table 11. Calculation of final correction factors $(K)$ for each $\mu \mathrm{s} / \mathrm{cm}$ setting on DSDP oscilloscope (data from Tables 7 through 10).

\begin{tabular}{lcccccc}
\hline Scope Setting $\mathrm{a}, \mathrm{b}, \mathrm{c}$ & \multicolumn{2}{c}{$1.0 \mu \mathrm{s} / \mathrm{cm}$} & \multicolumn{2}{c}{$2.0 \mu \mathrm{s} / \mathrm{cm}$} & \multicolumn{2}{c}{$5.0 \mu \mathrm{s} / \mathrm{cm}$} \\
Thickness of Semi-standards & $2.54 \mathrm{~cm}$ & $5.00 \mathrm{~cm}$ & $\leq 2.54 \mathrm{~cm}$ & $5.00 \mathrm{~cm}$ & $\leq 2.54 \mathrm{~cm}$ & $5.00 \mathrm{~cm}$ \\
Water $(0.957 \mathrm{~cm})$ & - & - & $1.0081^{\mathrm{b}}$ & - & - & - \\
Water $(2.5 \mathrm{~cm})$ & - & - & - & - & $1.0094^{\mathrm{b}}$ & - \\
Lucite & - & - & $1.0048^{\mathrm{b}}$ & - & $1.0015^{\mathrm{c}}$ & $1.0059^{\mathrm{b}}$ \\
Brass & $1.0121^{\mathrm{b}}$ & - & $1.0134^{\mathrm{c}}$ & $1.0137^{\mathrm{b}}$ & $0.9939^{\mathrm{c}}$ & $1.0089^{\mathrm{c}}$ \\
Aluminum & $1.0095^{\mathrm{b}}$ & - & $1.0176^{\mathrm{c}}$ & $1.0112^{\mathrm{b}}$ & $1.0059^{\mathrm{c}}$ & $1.0046^{\mathrm{c}}$ \\
Ea. avg. $K=$ & $1.0108^{\mathrm{b}}$ & - & $\left(1.0064^{\mathrm{b}}\right)$ & $1.0125^{\mathrm{b}}$ & $\left(1.0094^{\mathrm{b}}\right)$ & $\left(1.0059^{\mathrm{b}}\right)$ \\
Mean $^{\mathrm{d}} K=$ & $1.0108^{\mathrm{b}}$ & $\left(+1.0095^{\mathrm{b}}\right)$ & $\left(+1.0077^{\mathrm{b}}\right)$ \\
\hline
\end{tabular}

The best precision of the data occurs when using the full range of the $\mu \mathrm{s} / \mathrm{cm}$ delay dial:

a Good use: 6-10 range.

b Fair use: 3-6 range.

c Poor use: $0-3$ range.

d These mean $K$ for each "microsecond per cm" setting of the DSDP oscilloscope were used to calculate all Leg 51 sonic data. These sets of data averages do not include data labeled by superscript "c."

2) Sonic log (bore-hole compensated system, $9.21 \mathrm{~cm}$ diameter); caliper; and gamma-ray log (GR) (unsuccessful) $)^{5}$.

3) Density log (bore-hole compensated) (CDL), 6.99 $\mathrm{cm}$ in diameter; caliper; and $\mathrm{GR}^{6}$ (successful). Logging speed was $8.63 \mathrm{~m} / \mathrm{min}$. Time constant was 3 seconds.

4) Induction log and 16 -inch $(40-\mathrm{cm})$ normal resistivity, $9.21 \mathrm{~cm}$ in diameter; and GR (successful). Logging speed was $12.2 \mathrm{~m} / \mathrm{min}$. Time constant was 4 seconds.

5) Guard $\log , 8.9 \mathrm{~cm}$ diameter, and neutron log (thermal neutron, single detector and centered, therefore, qualitative), GR (unsuccessful) ${ }^{5}$. Logging speed was $12.2 \mathrm{~m} / \mathrm{min}$. Time constant was 2 seconds.

6) Temperature log (successful).

The following two suites of Gearhart-Owen logging tools were attempted in Hole 462A (both semi-successful):

1) Neutron log (thermal neutron), single detector and no positioning devices (free, therefore qualitative),

\footnotetext{
5 These tools could not be lowered through a bad hole in order to reach the un-washedout portion of the hole.

6 The GR on the density log is of fset by about $0.30 \mathrm{~cm}$ higher than the density reading.
}

and GR (4.29-cm diameter) were run through the pipe, drill-collars ( $>6737 \mathrm{~m}$ ) and bumper subs (6074 to 6084 $\mathrm{m})$. Logging speed was $12.2 \mathrm{~m} / \mathrm{min}$. Time constant was 2 seconds.

2) Sonic log (bore-hole compensated system), caliper, and GR. Logging speed was $15.2 \mathrm{~m} / \mathrm{min}$. Time constant was 2 seconds.

The GR tool is run with each logging run for stratigraphic control. As an example, the GR also allows the density and velocity on two different logging runs to be correlated, as the depths are not accurate enough.

In general, when interpreting any of these logs, one should consult Lynch (1962) and a Gearhart-Owen manual to determine what precautions and data corrections are necessary, and to find the proper charts in various manuals and perform any needed corrections.

All of the above-given data, except the first temperature $\log$, are available at various scales in analog plots and on magnetic tape. The first temperature log was semi-successful, and we have only a photographic copy of unedited raw data, as the magnetic tape collected at the site was inadequate. (See the Site Summary for discussion of hole conditions, casing, and drill pipe.) 
Table 12. Geometric condition of Leg 61 cores as they were processed through the Analog GRAPE. ${ }^{\mathrm{a}}$

\begin{tabular}{|c|c|c|c|c|}
\hline Hole & Core & Section & $\begin{array}{c}\text { Interval } \\
(\mathrm{cm})\end{array}$ & $\begin{array}{c}\text { Geometric } \\
\text { Condition }\end{array}$ \\
\hline 462 & $1-35$ & - & - & A \\
\hline 462 & 36 & 1 & $0-65$ & A \\
\hline 462 & 36 & 1 & 65-117 & $C-3$ \\
\hline 462 & 36 & 1 & $117-150$ & A \\
\hline 462 & 36 & $2-3$ & - & A \\
\hline 462 & 36 & 4 & - & C-3 \\
\hline 462 & 37 & - & - & A \\
\hline 462 & 38 & 1 & - & $C-3$ \\
\hline 462 & 38 & $2-4$ & - & A \\
\hline 462 & 38 & 5 & - & $C-3$ \\
\hline 462 & 39 & - & - & A \\
\hline 462 & $40-48$ & - & - & $C-1$ \\
\hline 462 & 49 & $1 ?-2$ & - & C-2 \\
\hline 462 & 49 & $3-5$ & - & $\mathrm{C}-1$ \\
\hline 462 & 54 & $1 ?$ & - & $C-1$ \\
\hline 462 & 54 & 2 & $0-60$ & $C-1$ \\
\hline 462 & 54 & 2 & $60-71$ & C-3 \\
\hline 462 & 54 & 2 & $71-90$ & C-1 \\
\hline 462 & 54 & 3 & $0-40$ & C-2 \\
\hline 462 & 54 & 3 & $40-47$ & C-3 \\
\hline 462 & 54 & 3 & $47-113$ & $C-1$ \\
\hline 462 & 54 & 3 & $113-150$ & C-3 \\
\hline 462 & $55 ?$ & - & - & C-3 \\
\hline 462 & 56 & 1 & & $C-2$ \\
\hline 462 & 56 & 2 & - & C-3 \\
\hline 462 & 57 & 1 & - & $C-2$ \\
\hline 462 & 57 & 2 & $0-28$ & C-1 \\
\hline 462 & 57 & 2 & $28-60$ & C-2 \\
\hline 462 & 57 & 2 & $60-150$ & $C-1$ \\
\hline 462 & 57 & 3 & $0-60$ & C-2 \\
\hline 462 & 57 & 3 & $60-110$ & $C-1$ \\
\hline 462 & 58 & $1-4$ & - & $C-1$ \\
\hline 462 & 58 & 5 & $0-15$ & C-3 \\
\hline 462 & 58 & 5 & $15-150$ & C-1 \\
\hline 462 & 59 & 1 & - & C-1 \\
\hline 462 & 59 & 2 & - & C-2 \\
\hline 462 & $60-69$ & - & - & C-1 (out of liner) \\
\hline $462 \mathrm{~A}$ & $1-2$ & - & - & A \\
\hline $462 \mathrm{~A}$ & $3 ?-7 ?$ & - & - & C-1? \\
\hline $462 \mathrm{~A}$ & $\mathrm{H}-3$ & $1-3$ & - & $C-1$ \\
\hline $462 \mathrm{~A}$ & $\mathrm{H}-3$ & 4 & - & C-2 \\
\hline $462 \mathrm{~A}$ & 8 to $\mathrm{H}-4$ & - & - & $C-1$ \\
\hline $462 \mathrm{~A}$ & 9 & 1 & - & $C-1$ \\
\hline $462 \mathrm{~A}$ & 9 & $2-5$ & - & $\mathrm{C}-2$ \\
\hline $462 \mathrm{~A}$ & 9 & 6 & - & $C-1$ \\
\hline $462 \mathrm{~A}$ & 10 & 1 & - & C-1 \\
\hline $462 \mathrm{~A}$ & 10 & 2 & $0-25$ & C-3 \\
\hline $462 \mathrm{~A}$ & 10 & 2 & $25-68$ & $C-2$ \\
\hline $462 \mathrm{~A}$ & 10 & 2 & $68-90$ & C-3 \\
\hline $462 \mathrm{~A}$ & 10 & 2 & $90-150$ & $\mathrm{C}-2$ \\
\hline $462 \mathrm{~A}$ & 10 & 3 & - & $C-1$ \\
\hline $462 \mathrm{~A}$ & 11 & 1 & $0-35$ & $C-1$ \\
\hline $462 \mathrm{~A}$ & 11 & 1 & $35-150$ & $C-2$ \\
\hline $462 \mathrm{~A}$ & 12 & - & - & C-2 \\
\hline $462 \mathrm{~A}$ & 13 & - & - & $C-1$ \\
\hline $462 \mathrm{~A}$ & $14-42$ & - & - & C-1 (out of liner) \\
\hline $462 \mathrm{~A}$ & 43 & - & - & $\mathrm{C}-1$ (in liner) \\
\hline $462 \mathrm{~A}$ & $44-74$ & - & - & C-1 (out of liner) \\
\hline $462 \mathrm{~A}$ & 74?-91? & - & - & C-1? (out of liner)? \\
\hline
\end{tabular}

a Code $\mathrm{A}=$ Soft sediment in liner: Soft sediment completely filled the core liner, and no diameter adjustments are necessary.

Code $\mathrm{C}=$ Hard rock in liner: Hard rock in liner, and it is noted (by code) whether the rock is surrounded (with "corrected density"):

Code 1 = air, $0.0 \mathrm{~g} / \mathrm{cm}^{3}$

Code 2 = water, $1.1 \mathrm{~g} / \mathrm{cm}^{3}$

Code 3 = sediment, $1.6 \mathrm{~g} / \mathrm{cm}^{3}$

Code 4 = breccia, $1.8 \mathrm{~g} / \mathrm{cm}^{3}$

If rock is surrounded by water or air, then the diameter is offset from the gamma ray beam axis $=3.305 \mathrm{~cm}$ - radius of the rock.

b The material surrounding the cores at this site was not recorded consistently onboard ship (because many people were involved); thus, this type of information is susceptible to errors. Therefore, if any density data appear anomalous, a future investigator may wish to attempt to recalculate densities by using different "geometric conditions" as models. The parameters that are listed here were reconstructed from photographs and used in the computer program.
Table 13. Apparent attenuation coefficients ${ }^{\mathrm{a}}$ determined from the $6.61-\mathrm{cm}$ and $2.54-\mathrm{cm}$ aluminum standards.

\begin{tabular}{|c|c|c|c|c|}
\hline $\begin{array}{c}\text { Aluminum, } \\
2.54 \mathrm{~cm} \\
\left(\mathrm{~cm}^{2} / \mathrm{g}\right)\end{array}$ & $\begin{array}{c}\text { Aluminum, } \\
6.61 \mathrm{~cm} \\
\left(\mathrm{~cm}^{2} / \mathrm{g}\right)\end{array}$ & Hole & $\begin{array}{c}\text { Between } \\
\text { Cores }\end{array}$ & Date \\
\hline 0.1093 & 0.1073 & 462 & $0-1$ & 22 May 1978 \\
\hline 0.1098 & 0.1077 & 462 & $0-1$ & 22 May 1978 \\
\hline 0.1088 & 0.1070 & 462 & $0-1$ & 22 Маy 1978 \\
\hline 0.1100 & 0.1076 & 462 & $0-1$ & 22 May 1978 \\
\hline 0.1083 & 0.1078 & 462 & $0-1$ & 22 May 1978 \\
\hline 0.1102 & 0.1077 & 462 & $0-1$ & 22 May 1978 \\
\hline 0.1096 & 0.1074 & 462 & $0-1$ & 22 May 1978 \\
\hline 0.1100 & 0.1077 & 462 & $0-1$ & 22 May 1978 \\
\hline 0.1105 & 0.1079 & 462 & $0-1$ & 22 May 1978 \\
\hline 0.1092 & 0.1079 & 462 & $0-1$ & 22 May 1978 \\
\hline 1.1096 & 0.1075 & 462 & $0-1$ & 22 May 1978 \\
\hline 0.1101 & 0.1079 & 462 & $0-1$ & 22 May 1978 \\
\hline 0.1098 & 0.1078 & 462 & $0-1$ & 22 May 1978 \\
\hline 0.1099 & 0.1078 & 462 & $0-1$ & 22 May 1978 \\
\hline 0.1092 & 0.1078 & 462 & $0-1$ & 22 May 1978 \\
\hline 0.1098 & 0.1079 & 462 & $0-1$ & 23 May 1979 \\
\hline 0.1097 & 0.1078 & 462 & $0-1$ & 23 May 1979 \\
\hline 0.1096 & 0.1077 & 462 & $0-1$ & 23 May 1979 \\
\hline 0.1099 & 0.1074 & 462 & $0-1$ & 23 May 1979 \\
\hline 0.1097 & 0.1079 & 462 & $0-1$ & 23 May 1979 \\
\hline 0.1108 & 0.1076 & 462 & $32-32$ & 28 May 1978 \\
\hline 0.1110 & 0.1077 & 462 & $35-35$ & 1 June 1978 \\
\hline 0.1103 & 0.1077 & 462 & $44-44$ & 2 June 1978 \\
\hline 0.1100 & 0.1084 & 462 & $48-48$ & 2 June 1978 \\
\hline 0.1105 & 0.1086 & 462 & $52-53$ & 3 June 1978 \\
\hline 0.1108 & 0.1084 & 462 & $52-53$ & 3 June 1978 \\
\hline 0.1108 & 0.1085 & 462 & $52-53$ & 3 June 1978 \\
\hline 0.1101 & 0.0085 & 462 & $52-53$ & 3 June 1978 \\
\hline 0.1107 & 0.1087 & 462 & $52-53$ & 3 June 1978 \\
\hline 0.1109 & 0.1082 & 462 & $54-55$ & 3 June 1978 \\
\hline 0.1097 & 0.1081 & 462 & $60-62$ & 4 June 1978 \\
\hline 0.1097 & 0.1078 & 462 & $60-61$ & 4 June 1978 \\
\hline 0.1096 & 0.1084 & 462 & $68-69$ & 5 June 1978 \\
\hline 0.1110 & 0.1082 & $462 \mathrm{~A}$ & $3-8$ & 10 June 1978 \\
\hline 0.1115 & 0.1082 & $462 \mathrm{~A}$ & $14-15$ & 11 June 1978 \\
\hline 0.1101 & 0.1081 & $462 \mathrm{~A}$ & $17-18$ & 12 June 1978 \\
\hline 0.1113 & 0.1084 & $462 \mathrm{~A}$ & $28-29$ & 18 June 1978 \\
\hline 0.1106 & 0.1081 & $462 \mathrm{~A}$ & $31-32$ & 21 June 1978 \\
\hline 0.1101 & 0.1085 & $462 \mathrm{~A}$ & $38-39$ & 22 June 1978 \\
\hline 0.1100 & 0.1085 & $462 \mathrm{~A}$ & $51-52$ & 26 June 1978 \\
\hline 0.1118 & 0.1086 & $462 \mathrm{~A}$ & $52-53$ & 26 June 1978 \\
\hline 0.1113 & 0.1081 & $462 \mathrm{~A}$ & $61-62$ & 28 June 1978 \\
\hline 0.1114 & 0.1081 & $462 \mathrm{~A}$ & $65-66$ & 30 June 1978 \\
\hline 0.1106 & 0.1080 & $462 \mathrm{~A}$ & $74-75$ & 8 July 1978 \\
\hline 0.1110 & 0.1082 & $462 \mathrm{~A}$ & $74-75$ & 8 July 1978 \\
\hline 0.1115 & 0.1083 & $462 \mathrm{~A}$ & $74-75$ & 8 July 1978 \\
\hline 0.1112 & 0.1081 & $462 \mathrm{~A}$ & $74-75$ & 8 July 1978 \\
\hline 0.1111 & 0.1083 & $462 \mathrm{~A}$ & $74-75$ & 8 July 1978 \\
\hline 0.1118 & 0.1080 & $462 \mathrm{~A}$ & $74-75$ & 8 July 1978 \\
\hline 0.1107 & 0.1079 & $462 \mathrm{~A}$ & $74-75$ & 8 July 1978 \\
\hline 0.1119 & 0.1080 & $462 \mathrm{~A}$ & $74-75$ & 8 July 1978 \\
\hline 0.1110 & 0.1076 & $462 \mathrm{~A}$ & $74-75$ & 8 July 1978 \\
\hline 0.1113 & 0.1081 & $462 \mathrm{~A}$ & $74-75$ & 8 July 1978 \\
\hline$\Sigma=5.8491$ & 5.7238 & & & \\
\hline \multicolumn{5}{|l|}{ No. $=53$} \\
\hline Avg. $=0.11036$ & 0.10799 & & & \\
\hline
\end{tabular}

${ }^{a}$ Apparent quartz attenuation coefficient $=\frac{\ln I_{\mathrm{O}} / I}{d\left(2.60 \mathrm{~g} / \mathrm{cm}^{3}\right)}$, where

$I_{\mathrm{O}}=$ gamma count through air, 2-minute period;

$I$ = gamma count through aluminum standard, 2 -minute period; $d=$ thickness of standard $(6.61 \mathrm{~cm}$ or $2.54 \mathrm{~cm})$. 
Table 14. Shipboard density determination for a calcite crystal (density $=2.715 \mathrm{~g} / \mathrm{cm}^{3}$ ) with the OH.AUS Centogram triple-beam balance $(311 \mathrm{~g})$.

\begin{tabular}{ccccc}
\hline & $\begin{array}{c}\text { Weight in } \\
\text { Air } \\
(\mathrm{g})\end{array}$ & $\begin{array}{c}\text { Weight in } \\
\text { Water } \\
(\mathrm{g})\end{array}$ & $\begin{array}{c}\text { Volume } \\
\left(\mathrm{cm}^{3}\right)\end{array}$ & $\begin{array}{c}\text { Density } \\
\left(\mathrm{g} / \mathrm{cm}^{3}\right)\end{array}$ \\
\hline 1 & 77.08 & 48.68 & 28.40 & 2.714 \\
2 & 77.09 & 48.66 & 28.43 & 2.712 \\
3 & 77.07 & 48.67 & 28.40 & 2.714 \\
4 & 77.06 & 48.67 & 28.39 & 2.714 \\
5 & 77.07 & 48.69 & 28.38 & 2.716 \\
6 & 77.08 & 48.68 & 28.40 & 2.714 \\
7 & 77.07 & 48.67 & 28.40 & 2.714 \\
8 & 77.07 & 48.64 & 28.43 & 2.711 \\
9 & 77.08 & 48.66 & 28.42 & 2.712 \\
10 & 77.06 & 48.65 & 28.41 & 2.712 \\
\multicolumn{4}{c}{ Mean $=$} & 2.713 \\
\hline
\end{tabular}

Table 15. Shipboard density determination for a basalt minicore $^{\mathrm{a}}$ with the OHAUS centogram triple-beam balance $(311 \mathrm{~g})$.

\begin{tabular}{ccccc}
\hline Weighing & $\begin{array}{c}\text { Weight in } \\
\text { Air } \\
(\mathrm{g})\end{array}$ & $\begin{array}{c}\text { Weight in } \\
\text { Water } \\
(\mathrm{g})\end{array}$ & $\begin{array}{c}\text { Volume }^{\mathrm{b}} \\
\left(\mathrm{cm}^{3}\right)\end{array}$ & $\begin{array}{c}\text { Wet-Bulkc } \\
\text { Density } \\
\left(\mathrm{g} / \mathrm{cm}^{3}\right)\end{array}$ \\
\hline 1 & 34.24 & 22.35 & 11.89 & 2.880 \\
2 & 34.23 & 22.36 & 11.87 & 2.884 \\
3 & 34.24 & 22.35 & 11.89 & 2.880 \\
4 & 34.24 & 22.35 & 11.89 & 2.880 \\
5 & 34.24 & 22.35 & 11.89 & 2.880 \\
6 & 34.24 & 22.36 & 11.88 & 2.882 \\
7 & 34.23 & 22.35 & 11.89 & 2.879 \\
8 & 34.24 & 22.35 & 11.89 & 2.880 \\
9 & 34.24 & 22.35 & 11.89 & 2.880 \\
10 & 34.24 & 22.36 & 11.88 & 2.882 \\
& \multicolumn{4}{c}{ Mean $=$} \\
\hline
\end{tabular}

a Sample 462-62-1, 85-87 cm

b Volume $=$ (weight in air minus weight in water $) \div 1.00$ $\mathrm{g} / \mathrm{cm}^{3}$.

c Wet-bulk density $=$ weight in air $\div$ volume .

\section{Natural Gamma Radiation (various-diameter gamma-ray logs)}

In general the GR log has a scintillation detector which is $15.2 \mathrm{~cm}$ long. The GR data are in American Petroleum Institute (API) units (American Petroleum Institute, 1959). The GR detects natural gamma radiation, emitted primarily from potassium, thorium series elements, and uranium-radium series elements in the formation. As a generality, carbonate and sandstone, without potassium feldspars, emit very little natural gamma radiation compared to clay and shale formations, which absorb radioactive isotopes as ions. Therefore, the GR is primarily used to distinguish shale and non-shale formations. The GR tool, interpretation problems, and data characteristics, such as time constant, logging speed, etc., are discussed in Lynch (1962) and Kokesh (1951).

\section{Sound Velocity}

During Leg 61, we ran the sonic log (bore-hole compensated system). This tool measures compressional- sound velocity in units of micro-seconds per foot ( 30.5 $\mathrm{cm})$. The basic principle of the tool and basic data-interpretation characteristics are described in Kokesh et al. (1965), Morris et al. (1963); in addition, "noise," "cycle skipping," and precautions in data interpretation are discussed in Lynch (1962). On the BHC tool, the two receivers were separated by $61 \mathrm{~cm}$ (vertical resolution), and the distance from the transmitter to the first receiver was $91.4 \mathrm{~cm}$.

The tool had a centering device-caliper; thus, the tool may have had too much stand-off from the side of the hole to be accurate (Lynch, 1962) for the low formation velocities involved.

Since the compensated sonic log is essentially a "twolayer seismic refraction experiment," and with the given geometry of the tool (centralized), when the formation's velocity approaches that of the fluid in the hole velocities below 1.7 to $1.8 \mathrm{~km} / \mathrm{s}$ probably are not measurable.

Velocities below 1.7 to $1.8 \mathrm{~km} / \mathrm{s}$ are severely affected by tool standoff (Fig. 2A) (distance of transmitters and receivers from bore-hole wall). For example, the chart in Figure 2B shows that the diameter of the hole must be less than that maximum diameter if that corresponding formation velocity is to be measured.

This diagram clearly indicates that we cannot measure a formation velocity less than $1.67 \mathrm{~km} / \mathrm{s}$ where the hole is $>12$ inches $(30 \mathrm{~cm})$ in diameter. Any velocities less than $1.67 \mathrm{~km} / \mathrm{s}$ are partially or completely those of the fluid in the bore hole.

\section{Formation Wet-Bulk Density}

Leg 61 used the density (bore-hole compensated) log. The source and two receivers were held against the side of the hole by a motorized eccentralizer, which also acts as a caliper. The density log is calibrated for density in limestone matrix, with fresh water in pores.

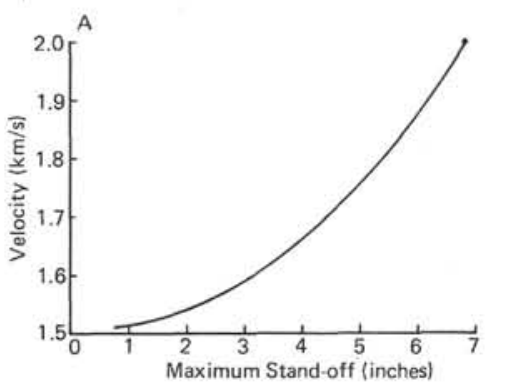

Lynch (1962) p. 275

$$
S=\left[\frac{1}{2}\left(\frac{1-\beta}{1+\beta}\right)^{1 / 2}\right]
$$

Where:

$S=$ Standoff of sonic transmitter and receiver

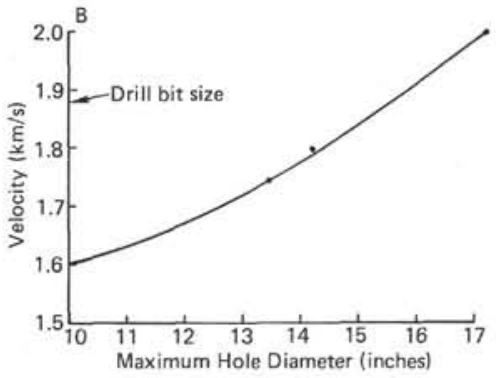

$1=$ Distance from transmitter to first receiver

$\beta=$ Ratio of hole-fluid velocity to formation velocity

Assumes compensated sonic tool with $I=3$ feet and tool diameter of 3.5 inches.

Figure 2. A. Maximum possible tool stand-off if the corresponding formation velocity is to be measured. B. Mazimum possible hole diameter in measuring the corresponding formation velocity. 
The tool measures an approximate wet-bulk density using a gamma-ray back-scattering technique. Wet-bulk density is the ratio of the "mass of wet-saturated (if in situ gas is not present) formation" to its "volume," expressed as grams per cubic centimeter. The general principles of this technique, similar logging tools, and their interpretation characteristics, assumptions, and precautions are discussed in Wahl et al. (1964) and Sherman and Locke (1975). Additional discussion of general principles is in Baker (1957) and Lynch (1962). Vertical resolution and penetration are about $30 \mathrm{~cm}$, depending on formation density and logging speed. The GR on the density $\log$ is offset by about $0.30 \mathrm{~cm}$ higher than the density reading.

\section{Formation Porosity (neutron tool)}

The neutron tool has a single detector, it is free (not positioned in the hole); thus, it is a qualitative tool. It emits thermal neutrons which are primarily (but not completely, e.g., $\mathrm{Cl}$ ) attenuated by collisions with $\mathrm{H}^{+}$ protons (same mass as neutrons) in the pore water. With the proper matrix correction, the tool qualitatively is an indicator of porosity. The tool's principal assumptions and precautions are discussed in Tittle (1961) and Lynch (1962). The depth of investigation and vertical resolution is on the order of $33 \mathrm{~cm}$, depending on logging speed, porosity, and type of formation.

\section{Electric-Logging Tools}

The induction log (six-coil system; 20,000 cycles/s) has a $100-\mathrm{cm}$ vertical resolution, and about $50 \%$ of the signal has an approximate $155-\mathrm{cm}$ radial depth of investigation; the 16-in $(40.6-\mathrm{cm})$ normal tool has approximately a $40.6-\mathrm{cm}$ vertical resolution and radial depth of investigation. The induction tool is designed primarily to measure electrical conductivity accurately in highporosity sediment and rock (with resistivity $<100 \mathrm{ohm}$ $\mathrm{m})$. The guard log (Laterolog-3) is a focused electricalresistivity tool designed with a $15-\mathrm{cm}$ vertical resolution, and $50 \%$ of the signal has approximately $65 \mathrm{~cm}$ of radial penetration. It is designed to accurately measure electrical resistivity of hard rocks (such as basalt with $>100$ ohm-m resistivity). All of these electrical tools are affected by the geometry and resistivity of the formations and borehole. Therefore, the caliper data should be used from the density log to properly correct these electric logs to true formation resistivities. See Lynch (1962), Doll (1949, 1951), Moran and Kunz (1962), Tixier et al. (1959), and Keller and Frischnecht (1966) for detailed discussions and interpretation precautions.

\section{Temperature Log}

On Leg 61, we used the differential and absolute temperature log, which is $4.65 \mathrm{~cm}$ in diameter and has a thermocouple which records temperature continuously versus depth. Its precision is better than $\pm 0.05^{\circ} \mathrm{C}$, with an accuracy of \pm 2 or $3 \%$ of the calibrated range. The tool has a platinum-tip resistance thermometer. Temperature tools in general are briefly described in Lynch (1962).
The differential tool compares the temperatures immediately being measured with an earlier-measured temperature (measured with same element), but stored in a memory bank, from which the value is recalled after a given time. The difference in these two temperature measurements is plotted versus depth.

\section{Uyeda Temperature Probe}

The Uyeda temperature probe has a long $(\sim 30 \mathrm{~cm})$ and thick $(\sim 3 \mathrm{~cm})$ metal probe that is inserted into the bottom of the drill-hole via attachments through the drill bit. In the $\sim 3-\mathrm{cm}$-diameter metal tip is a thin $(\sim 0.5 \mathrm{~cm})$ thermistor. On Leg 61 , this tool was adjusted to automatically record the temperature every 2 minutes. With this tool, it is possible to record 128 data points over a total period of 256 minutes. Therefore, the data are in the form of time versus temperature. For example, the tool is lowered through the pipe, passing the ocean-water column and passing the hole while recording temperature (every $2 \mathrm{~min}$ ) on the way down; in addition, while the probe is inserted into the bottom of the hole, where it remains until temperature equilibrium has obtained maximum temperature after $\sim 20$ minutes, the probe is removed and pulled out of the hole while still recording temperature every 2 minutes. The oceanbottom water temperature is measured on the way up (the tool stops at $\sim 20$ meters above sea floor for $\sim 10$ min). By plotting time versus temperature we can recognize the temperature measurements in the ocean-water column, the sediment at the bottom of the hole, and the ocean-bottom water 20 meters above the sea floor.

The temperature tool has a theoretical precision and accuracy of $\pm 0.05^{\circ} \mathrm{C}$.

We attempted six temperature measurements, whose results are listed in Table 16, and the temperature-versus-time plots and interpretations for each attempt are in Figures 3 through 8.

Of the results, the first three runs appear to be the more-reliable data. Runs 4 through 6 were done with a different thermistor; thus, they may not be completely compatible with the first three runs. Run 6 has an anomalous ocean-bottom water temperature; thus, the sediment temperatures probably are not valid.

Table 16. Uyeda temperature probe results for Hole 462.

\begin{tabular}{cccc}
\hline $\begin{array}{c}\text { Run } \\
\text { Number }\end{array}$ & $\begin{array}{c}\text { Depth below } \\
\text { Sea Floor } \\
(\mathrm{m})\end{array}$ & $\begin{array}{c}\text { Sediment } \\
\text { Temperature } \\
\left({ }^{\circ} \mathrm{C}\right)\end{array}$ & $\begin{array}{c}\text { Sea Water } \\
\text { Temperature } \\
\left({ }^{\circ} \mathrm{C}\right)\end{array}$ \\
\hline 1 & 133.5 & 8.4 & 1.9 \\
2 & 181.0 & 10.7 & 1.9 \\
3 & 219.0 & 12.3 & 1.9 \\
4 & 257.0 & 15.9 & $2.0^{\mathrm{a}}$ \\
5 & 295.0 & $18.9(14.9)^{\mathrm{b}}$ & $2.1^{\mathrm{a}}$ \\
6 & 314.5 & 13.6 & $4.3^{\mathrm{a}}$ \\
\hline
\end{tabular}

${ }^{a}$ Measurement going in; temperature measurements on these runs used a different thermistor and may not be reliable; run 6 appears to be anomalous.

b Temperature at first plateau. 


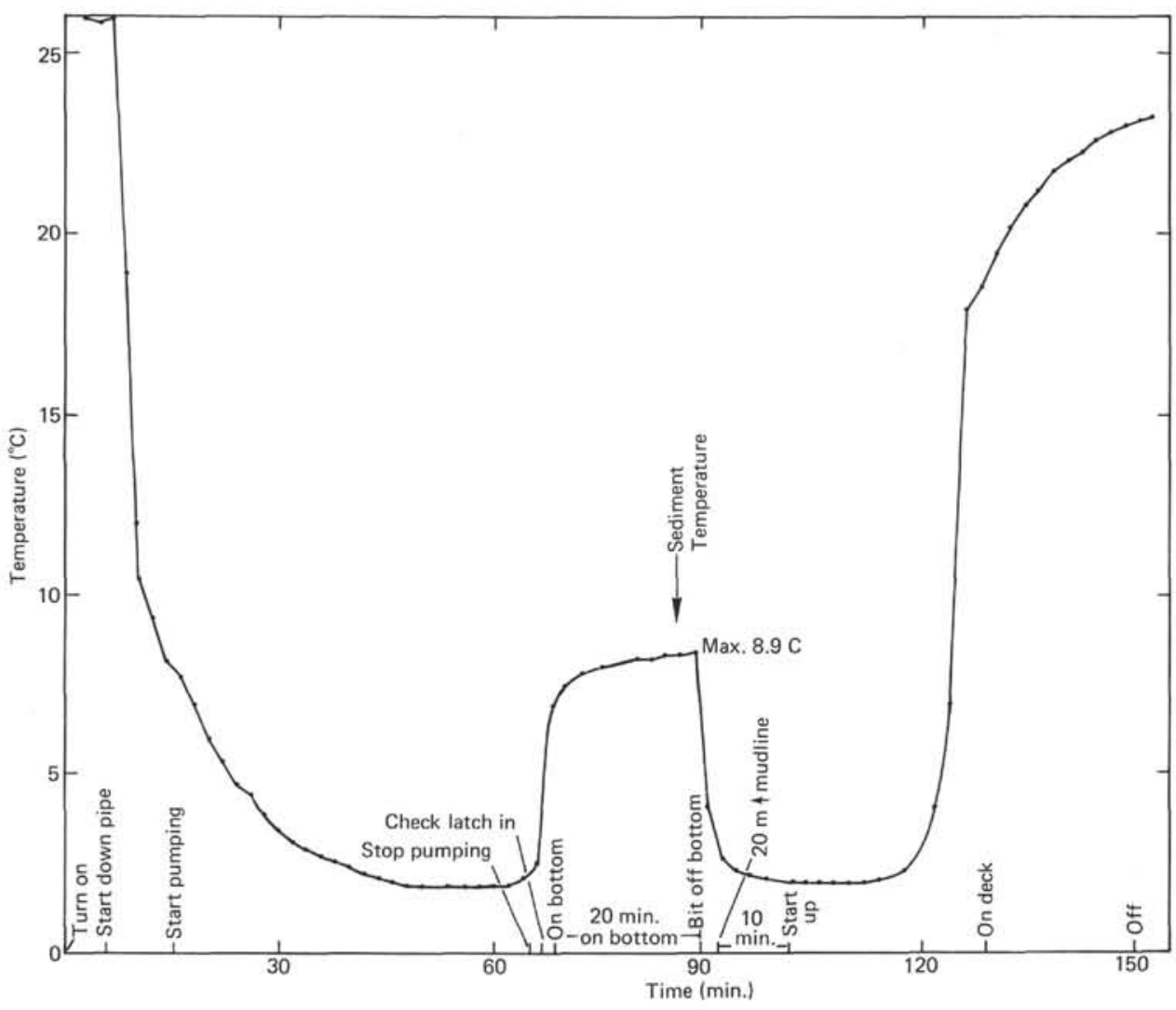

Figure 3. Time versus temperature for run 1 of the Uyeda temperature probe. Results are in Table 13.

\section{REFERENCES}

American Petroleum Institute, 1959. Recommended Practice for Standard Calibration and Form for Nuclear Logs. Am. Petrol. Inst. RP, 33.

Baker, P. E., 1957. Density logging with gamma rays. Trans. AIME, 210:289-294.

Boyce, R. E., 1973. Appendix I. Physical properties summary. In Edgar, N. T., Saunders, J. B., et al., Init. Repts. DSDP, 15: Washington (U.S. Govt. Printing Office), 1067-1068. 1976a. Definitions and laboratory techniques of the compressional sound velocity parameters and wet-water content, wetbulk density, and porosity parameters by gravimetric and gamma ray attenuation techniques. In Schlanger, S. O., Jackson, E. D., et al., Init. Repts. DSDP, 33: Washington (U.S. Govt. Printing Office), 931-958.

,1976b. Deep Sea Drilling Project procedures for shear strength measurement of clayey sediment using Modified Wykeham Farrance Laboratory Vane Apparatus. In Barker, P. F., Dalziel, I. W. D., et al., Init. Repts. DSDP, 36: Washington (U.S. Govt. Printing Office), 1059-1068.

Clark, S. P., Jr., 1966. Thermal conductivity. In Clark, S. P., Jr. (Ed.), Handbook of Physical Constants: Geol. Soc. Am. Mem., 97:459-482.

Doll, H. G., 1949. Introduction of induction logging and application to logging of wells drilled with oil base mud. Trans. AIME, 186: $148-164$.

1951. The Laterolog: a new resistivity logging method with electrodes using an automatic focusing system. Trans. AIME, 192: 305-316.

Evans, H. B., 1965. GRAPE-a device for continuous determination of material density and porosity. Transactions of the Sixth Annual SPWIA Logging Symposium, Dallas, Texas (Vol. 2), B1-B25.

Keller, G. V., and Frischknecht, F. C., 1966. Electrical Methods in Geophysical Prospecting: New York (Pergamon Press).

Kokesh, F. P., 1951. Gamma-ray logging. Oil Gas J., 50:284-290.
Kokesh, F. P., Schwartz, R. J., Wall, W. B., et al., 1965. A new approach to sonic logging and other acoustic measurements. $J$. Petrol. Technol., 17:282-286.

Lynch, E. J., 1962. Formation Evaluation: New York (Harper and Row).

Mattaboni, P., and Schreiber, E., 1967. Method of pulse transmission measurements for determining sound velocities. J. Geophys. Res., $72: 5160$.

Moran, J. H., and Kunz, K. S., 1962. Basic theory of induction logging and application to study of two-coil sondes. Geophys., $27: 829-858$.

Morris, R. L., Grine, D. R., and Arkfeld, T. E., 1963. The use of compressional and shear strength amplitudes for the location of fractures. Society of the Petroleum Engineers of AIME, 38th Annual Fall Meeting, New Orleans, Louisiana, 6-9 October, 1963. Paper number SPE 723, pp. 1-13.

Rocker, K., 1974. Physical properties and measurements and test procedures for Leg 27. In Veevers, J. J., Heirtzler, J. R., et al., Init. Repts. DSDP, 27: Washington (U.S. Govt. Printing Office), 1060.

Sherman, H., and Locke, S., 1975. Effect of porosity on depth of investigation of neutron and density sondes. Society of the Petroleum Engineers of AIME, 50th Annual Fall Meeting, Dallas, Texas, 28 September-1 October, 1975. Paper number SPE 5510, pp. 1-12.

Tittle, C. W., 1961. Theory of neutron logging. Geophys, , 26:27-29.

Tixier, M. P., Alger, R. P., and Tanghy, D. R., 1959. New developments in induction and sonic logging: Society of the Petroleum Engineers of AIME, 34th Annual Fall Meeting, Dallas, 4-7 October, 1959. Paper number 1300-G, pp. 1-18.

Von Herzen, R. P., and Maxwell, A. E., 1969. The measurement of thermal conductivity of deep-sea sediments by the needle-probe method. J. Geophys. Res., 64:1557.

Wahl, J. S., Tittman, J., and Johnstone, C. W., 1964. The dual spacing formation density log. J. Petrol. Technol., 16:1411-1416. 
R. E. BOYCE

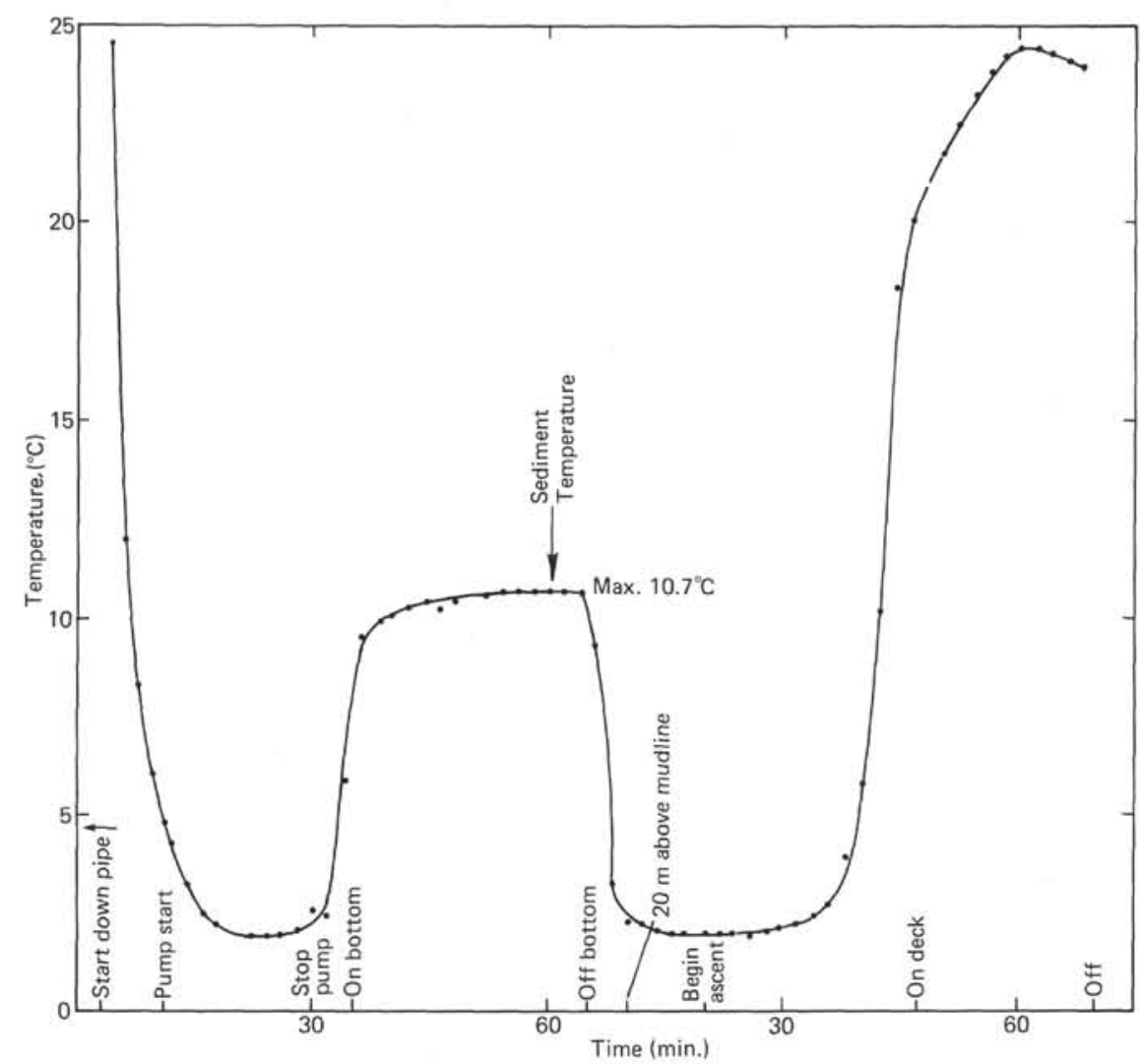

Figure 4. Time versus temperature for run 2 of the Uyeda temperature probe. Results are in Table 13.

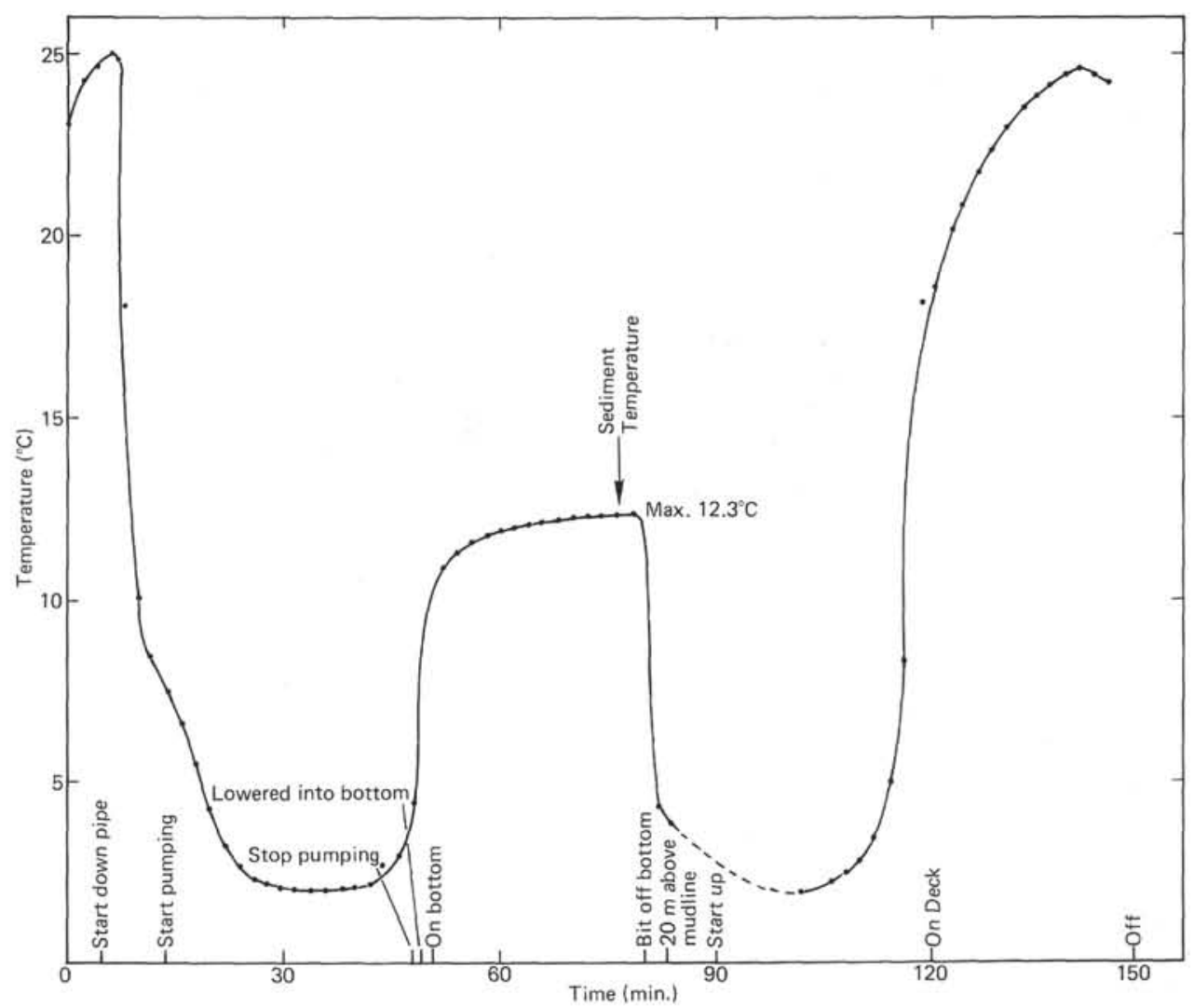

Figure 5. Time versus temperature for run 3 of the Uyeda temperature probe. Results are in Table 13. 


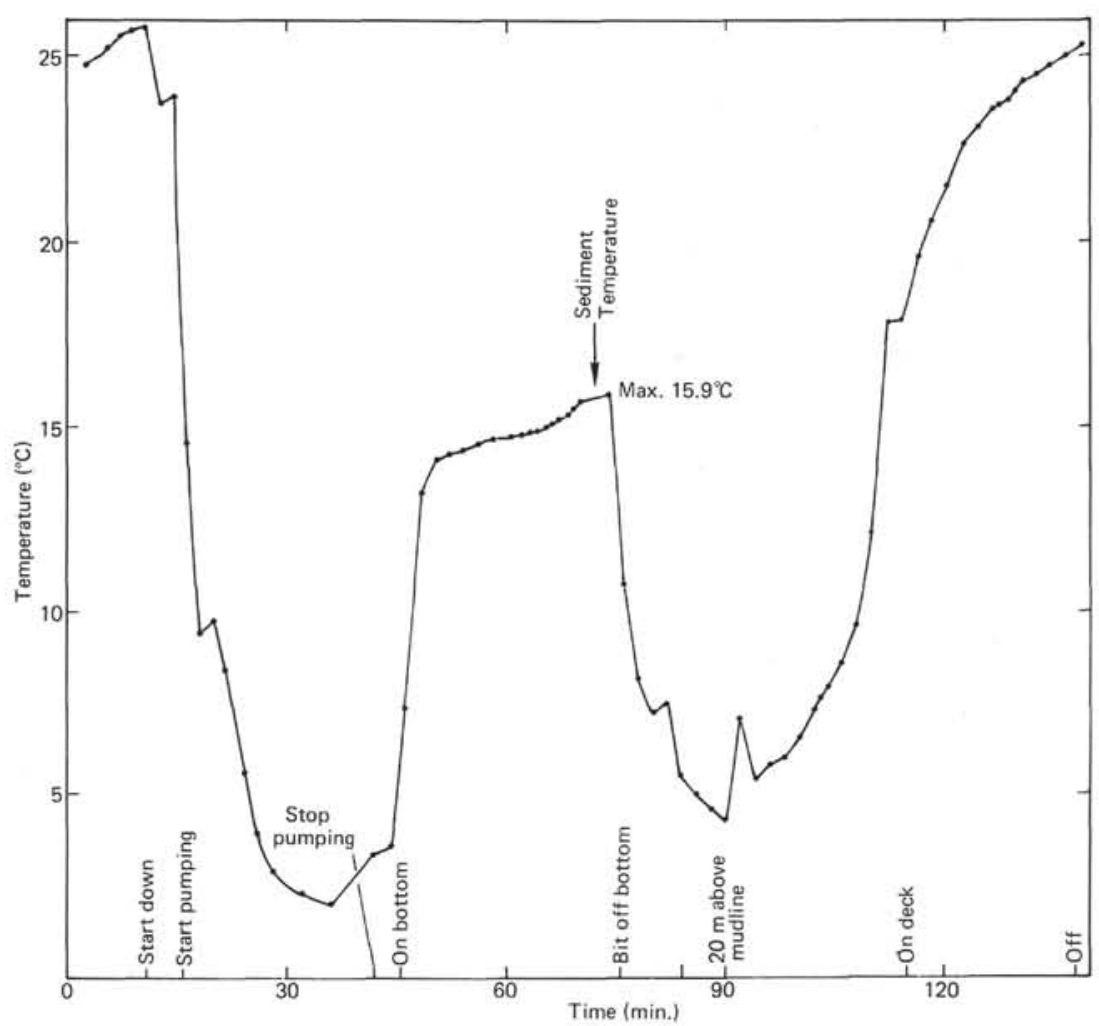

Figure 6. Time versus temperature for run 4 of the Uyeda temperature probe. Results are in Table 13.

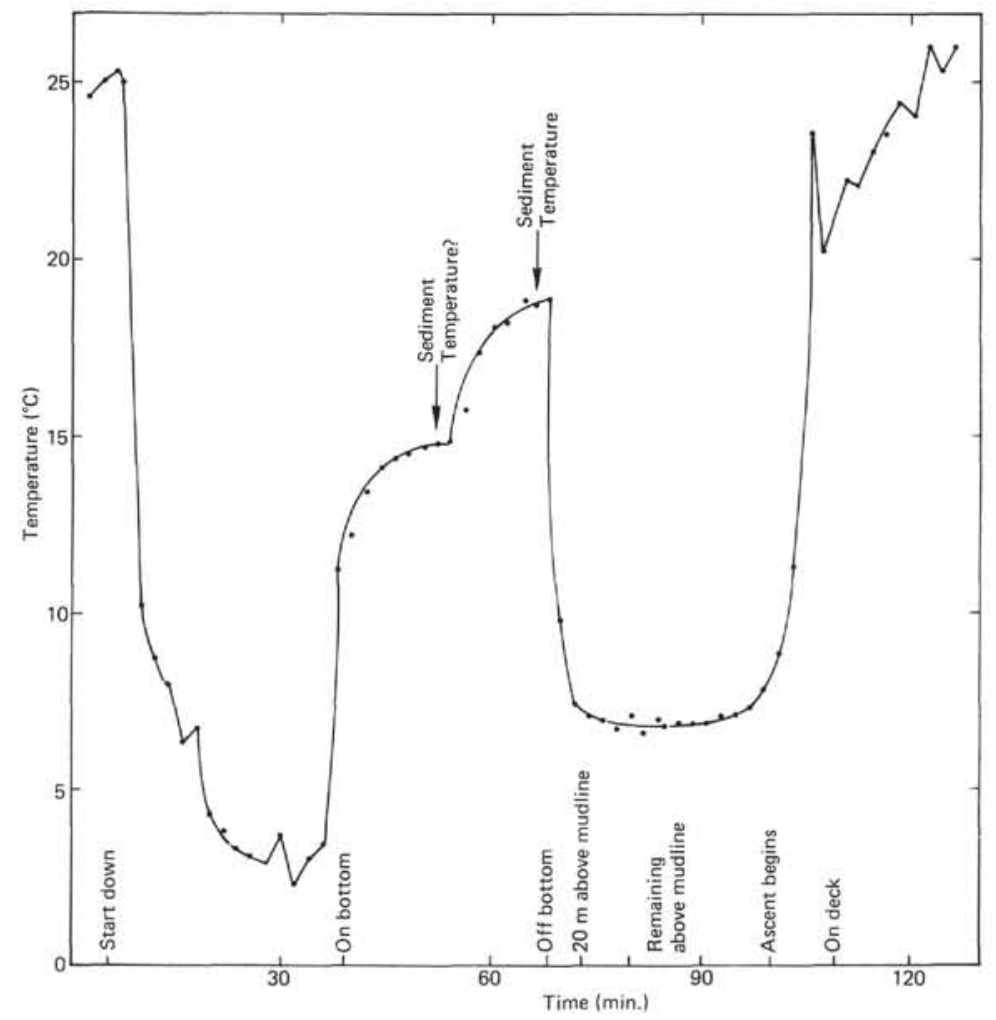

Figure 7. Time versus temperature for run 5 of the Uyeda temperature probe. Results are in Table 13. 
R. E. BOYCE

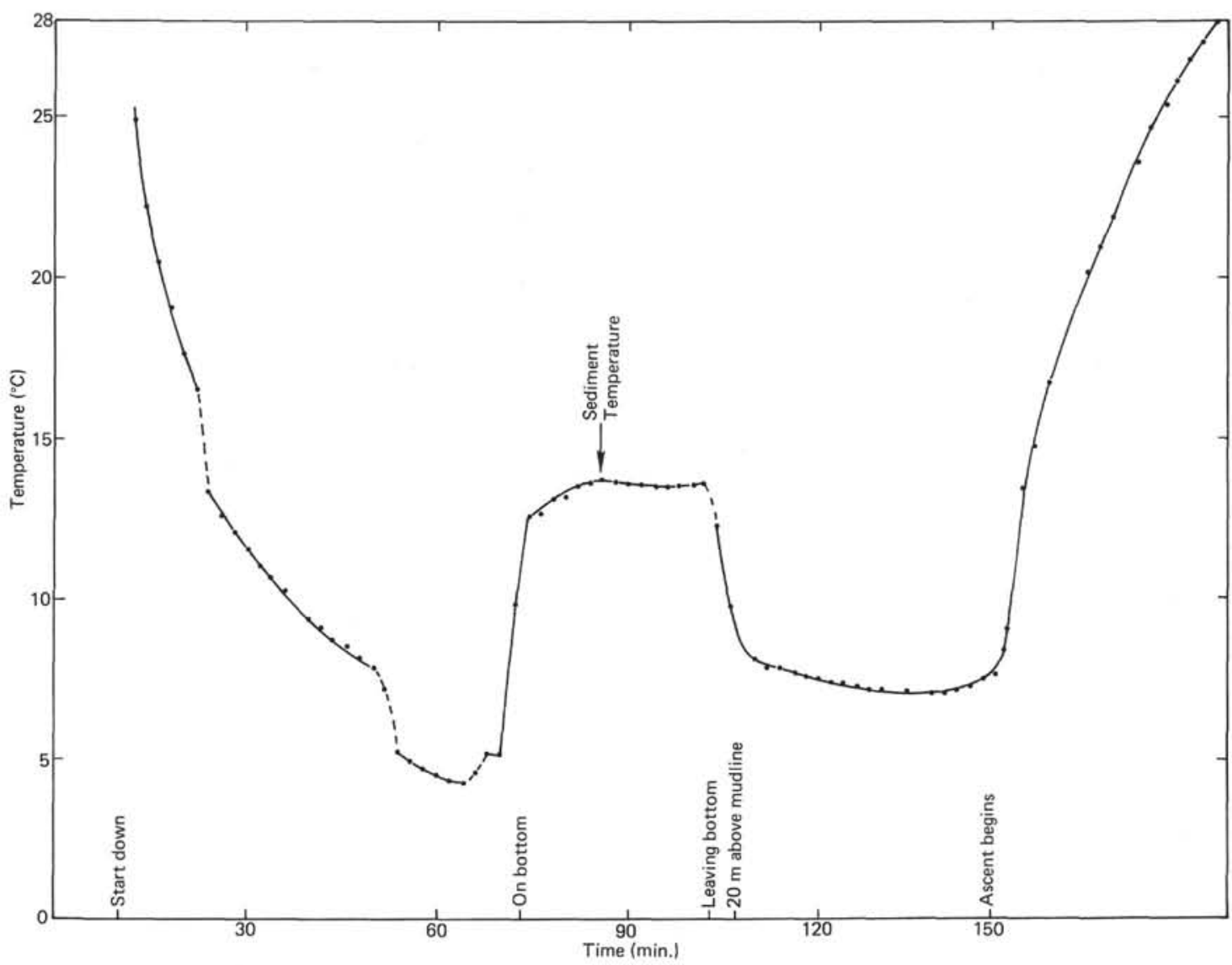

Figure 8. Time versus temperature for run 6 of the Uyeda temperature probe. Results are in Table 13. 